\title{
Chemical and Biochemical Engineering Approaches in Manufacturing Polyhydroxyalkanoate (PHA) Biopolyesters of Tailored Structure with Focus on the Diversity of Building Blocks
}

\author{
M. Koller a,b,* \\ aUniversity of Graz, Office of Research Management and Service, c/o \\ Institute of Chemistry, NAWI Graz, Heinrichstrasse 28/III, 8010 Graz \\ ${ }^{b}$ ARENA - Association for Resource Efficient and Sustainable \\ Technologies, Inffeldgasse 21b, 8010 Graz, Austria
}

doi: 10.15255/CABEQ.2018.1385 International License

Polyhydroxyalkanoates (PHA) constitute prokaryotic storage materials not only harnessing microbial cells with benefits for survival under challenging environmental conditions, but also attracting attention as biological materials with properties resembling those of currently used thermoplasts and elastomers of petrochemical origin. Strongly dependent on their monomeric composition and microstructure, PHA's exact material properties are predestined in statu nascendi, hence, during their biosynthesis. The present review sheds light on established and emerging strategies to produce differently composed PHA homo-, co-, ter-, and quarterpolyesters from the groups of short-, medium-, and long-chain PHA. It is shown how microbial strain selection, sophisticated genetic strain engineering based on synthetic biology approaches, advanced feeding strategies, and smart process engineering can be implemented to generate PHA of tailored monomeric composition, microstructure, molar mass, and molar mass distribution. Tailoring these parameters offers the possibility to produce customer-oriented PHA for various purposes, such as packaging materials, carriers of pharmaceutically active compounds, implants, or other emerging fields of use.

Keywords:

biopolyesters, heteropolyesters, homopolyesters, microstructure, monomers, polyhydroxyalkanoate (PHA)

\section{Introduction}

In almost every situation of our daily life, we are confronted with plastics; in the majority of cases, plastics are polymers of petrochemical origin. Over the last decades, they are surely the most emerging group of materials to produce custom-made items applied for packaging, and uses in the building industry, transportation, electronics, agriculture, healthcare, the sport sector, or leisure industry. However, we witness increasing global concern associated with petrochemical plastics. Valid estimations report that, over the last decades, a total of 8 to 9 gigatons (Gt) of plastics has been produced worldwide. ${ }^{1}$ Considering a current global plastic production approaching 400 megatons (Mt) per year, with an incredible upward trend, especially in emerging and developing countries, it is clear that the huge amounts of spent plastics, which already surpass $150 \mathrm{Mt}$ per year, have to be disposed

"Corresponding author: martin.koller@uni-graz.at Tel.: +43-316-380-5463 of somehow. ${ }^{2,3}$ Based on the high recalcitrance of these non-natural polymers, which prevents them from biodegradation, disposal strategies contemporarily in use are landfilling or simple release into the ecosphere (the fate of an estimated $79 \%$ of all plastics produced to date), or thermal conversion via incineration (estimated $12 \%$ of all plastics produced to date), all leading to unwanted consequences, such as growing piles of plastic waste or generation of toxic and/or greenhouse gas active gaseous emissions. ${ }^{1} \mathrm{~A}$ topical issue is the severe pollution of the marine environment by roughly $2 \mathrm{Mt}$ of plastic waste entering oceans via rivers, which, often as "microplastic", endangers the entire food chain. ${ }^{4}$ Recycling of petrochemical plastics only functions to a certain degree due to the need of a defined degree of purity of spent plastic, sorting accuracy, and decrease in material quality with each recycling cycle. ${ }^{2,5,6}$

As a real alternative, one can resort to solutions provided by Mother Nature. Originally found as light-refractive inclusion bodies in Bacillus megate- 
rium cells more than 90 years ago, polyhydroxyalkanoate (PHA) biopolyesters currently are in the scientific spotlight of microbiology, systems biology, synthetic biology, material scientists, and process engineers due to their versatile material features, which make them attractive for use in various sectors of the plastic market. ${ }^{7,8}$ From the microbiological point of view, it is more and more understood that the presence of PHA in microbial cells from the domains Bacteria and Archaea serves for protection of the cells against starvation and a range of other stress factors, which have been only recently described to be correlated with PHA biosynthesis. ${ }^{9}$ In this context, UV-irradiation, ${ }^{10}$ oxidative stress, ${ }^{11,12}$ hypertonic shock, ${ }^{13}$ freezing, ${ }^{14}$ or elevated temperature ${ }^{12}$ are described as stressors, which effects can be alleviated by the presence of PHA. Typically, PHA synthesis in microbes is favored by limitation of a nutrient essential for cell propagation, such as nitrogen or phosphate, in parallel to excessive availability of exogenous carbon source. Fig. 1 provides an electron microscope image of PHA-rich cells of the bacterium Cupriavidus necator cultivated in a continuously operated bioreactor cascade on glucose as carbon source.

Mainly the price of the latter determines the production price of PHA, which calls for the use of inexpensive carbon sources for large-scale PHA production. ${ }^{8}$ In this context, biorefinery concepts are currently in status of development to study the possibility of using carbon-rich side streams of different industrial processes as substrates for PHA

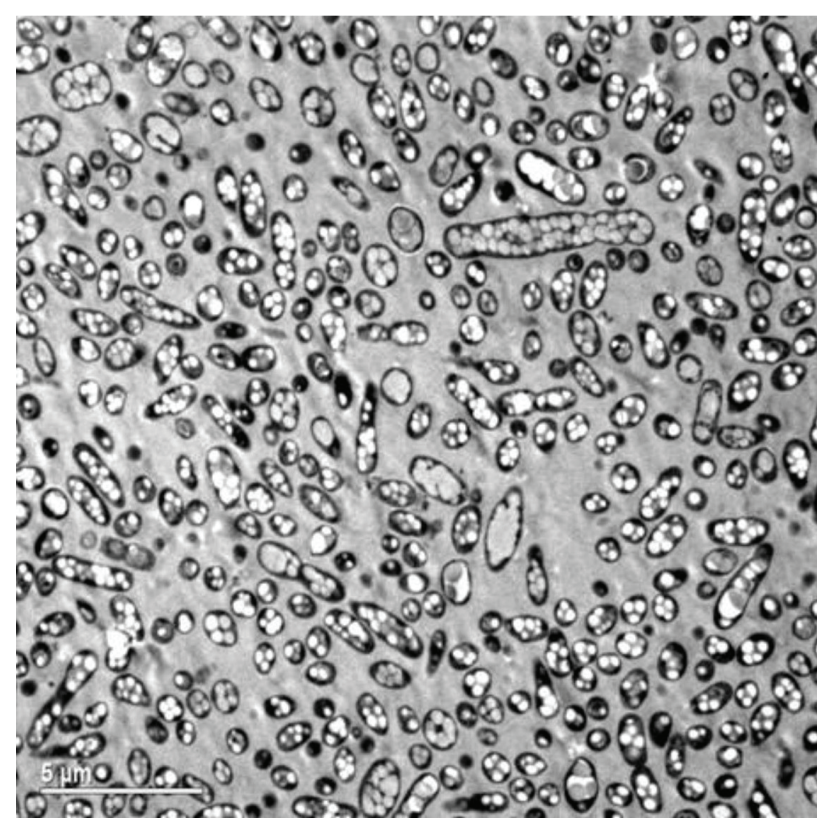

Fig. 1 - Electron microscope image of PHA-rich C. necator cells. The PHA content is well visible as white inclusion bodies ("carbonosomes"). Magnification: 20,000. Image kindly provided by E. Ingolić, FELMI-ZFE Graz. production. ${ }^{15,16}$ As an example, the coupling of PHA production to the rendering and animal processing industry was successfully demonstrated by feeding PHA-producing microbes low quality biodiesel fractions stemming from transesterification of animal-based waste lipids, crude glycerol, and offal materials. ${ }^{17}$ Other biorefinery concepts include PHA production from lignocellulose-rich waste like bagasse, wood or straw, ${ }^{18,19}$ side streams of the sugar production, ${ }^{20}$ the use of municipal wastewater, ${ }^{21}$ waste of the pulp and paper industry, ${ }^{22}$ surplus whey from dairy and cheese making processes, ${ }^{23}$ side streams of olive oil production, ${ }^{24}$ or $\mathrm{CO}_{2}$-rich effluent gas from power plants. ${ }^{25}$ In particular, the use of C1-compounds like $\mathrm{CO}_{2}$, syngas, or methanol for PHA production is currently attracting increasing attention. ${ }^{26-30}$

Beside the raw material aspect, downstream processing to recover PHA from microbial biomass in an efficient and environmentally sound fashion constitutes another crucial aspect of the PHA production chain. Here, solvent-based techniques for PHA extraction, enzymatic, chemical, and mechanical methods for disintegration of non-PHA biomass are exhaustively summarized in current review articles, all of these methods displaying shortcomings either in economic terms, environmental, and safety aspects, recovery efficiency, product purity, or scalability; this clearly indicates that PHA recovery displays a crucial aspect in making PHA market-fit. ${ }^{31-33}$ Emerging trends in optimizing PHA recovery encompass application of supercritical fluids, ${ }^{34-37}$ non-toxic solvents, ${ }^{38}$ the use of ionic liquids, ${ }^{39}$ and even the selective removal of non-PHA biomass via digestion by animals, such as the meal worm Tenebrio molitor. ${ }^{40}$ Moreover, genetic engineering of microbial strains is an emerging tool to facilitate PHA revovery. ${ }^{41}$

Regarding microbial strain selection, we currently witness an emerging trend towards robust organisms with a broad substrate spectrum and well understood metabolome, proteome and genome. In this context, the use of extremophiles isolated from challenging habitats, and halophile microbes isolated from salt lakes or the sea is strongly increasing these days in order to use them as work horses in simple, flexible, and stable cultivation setups, which can be operated in an energy-efficient manner due to the need for only restricted sterility precautions. ${ }^{42-46}$

Moreover, the use of different bioreactor systems, operated continuously or discontinuously, was exhaustively reviewed recently, indicating that bioreactor setup, process regime, and feeding strategy have major impact on PHA productivity, composition, and microstructure. ${ }^{47}$ 


\section{Different groups of PHA}

From the application-oriented point of view, it is pivotal to produce PHA not only in a cost-efficient way, but, to an increasing extent, to design PHA and follow-up products of properties and material quality competitive with the material performance of their petrochemical counterparts. During the last years, the number of PHA of different monomeric composition, biotechnologically produced both by microbial wild type strains and genetically engineered organisms, has increased considerably. Beside rather simple aliphatic PHA homo- and heteropolyesters (the latter encompassing co-, ter-, and quarterpolyesters) which were described decades ago, more recent reports describe the production of PHA harboring unsaturated, branched, and even aromatic building blocks, many of them displaying intriguing material features regarding the degree of crystallinity $\left(X_{c}\right)$, glass transition temperature $\left(T_{g}\right)$ as a measure for the mobility of polymer chains, melting temperature $\left(T_{m}\right)$, elongation at break (fracture strain, $\left.\varepsilon_{R}\right)$, tensile strength $\left(\sigma_{R}\right)$, and others. ${ }^{7,8}$ In order to encompass all characteristic aspects of PHA present at the same time in an investigated biological sample, the expression "PHAome" was recently coined by Chen and Hajnal. This terminus describes the diversity of monomeric PHA building blocks and their distribution, PHA homopolyesters, random and blocky structured PHA heteropolyesters, occurrence of intracellular PHA blends, and different molar masses of accumulated PHA; hence, the "PHAome" reflects the whole of different types of PHA in a microbial sample at a defined time point during biosynthesis. ${ }^{48}$ The present review provides an overview of described types of PHA, compares their material characteristics, and illustrates production strategies applied to produce them by implementing suitable strain-substrate-process regime combinations.

\section{The PHA enzymatic machinery}

\section{From substrates to PHA building blocks}

The principle enzymatic machinery needed for microbial PHA biosynthesis is already well explored and understood by the scientific community. ${ }^{49}$ In principle, carbon sources, such as sugars, fatty acids, glycerol, and others are converted to oxoacyl-CoA thioesters, most frequently to acetoacetyl-CoA generated by the 3-ketothiolase catalyzed condensation of the central metabolite acetyl-CoA. Now, the oxoacyl-CoA thioesters are reduced to the corresponding $(R)$-hydroxyacyl-CoA thioesters by the catalytic action of NADPH-dependent oxoacyl-CoA reductases; in analogy to ethanol production from the metabolic intermediate acetaldehyde by yeast, this step regenerates reducing equivalents; therefore, PHA biosynthesis can be considered as a "pseudo-fermentation" (reviewed by Braunegg et al.)..$^{50}$

\section{PHA synthases}

Finally, polyester synthases, as the key enzymes of PHA biosynthesis, come into action; they catalyze the conversion of $(R)$-hydroxyacyl-CoA thioesters to polyesters by releasing free CoA. In general, high intracellular pools of ATP, NAD $(\mathrm{P}) \mathrm{H}$, and acetyl-CoA and low levels of free CoA are favorable for the activity of enzymes responsible for PHA biosynthesis; such conditions occur, e.g., when the tricarboxylic acid cycle or other central metabolic pathways are blocked due to the absence of a growth-essential growth factor like, e.g., nitrogen or phosphate sources. ${ }^{50,51}$ Class I and class II PHA synthases comprise enzymes consisting of only one type of subunit (PhaC) with molecular masses between $61 \mathrm{kDa}$ and $73 \mathrm{kDa}$. According to their in vivo and in vitro substrate specificity, class I PHA synthases, e.g., the enzyme found in the best known PHA producer Cupriavidus necator, preferentially utilize various $(R)$-hydroxyacyl-CoA thioesters with the acyl group consisting of 3 to 5 carbon atoms. In contrast, class II PHA synthases (prototype organisms Pseudomonas aeruginosa or Pseudomonas putida) preferentially accept $(R)$-hydroxyacyl-CoA thioesters with the acyl group consisting of 6 to 14 carbon atoms as substrates. Such longer $(R)$-hydroxyacyl-CoA thioesters are generated by $\beta$-oxidation of the respective fatty acids. Here, it should be mentioned that the $\beta$-oxidation pathway first generates $(S)$-3-hydroxyacyl-CoA, which cannot be directly incorporated into $\mathrm{mcl}$-PHA due to stereospecificity of the $\mathrm{PhaC}$ subunit, which requires $(R)$-isomers as substrates. Therefore, $m c l$-PHA biosynthesis from lipids requires specific isomerases to convert the $(S)$ - into the $(R)$-isomers. Moreover, class III PHA synthases, such as those found in the prototype organism Allochromatium vinosum, encompass enzymes comprising of two different subunits, namely, the PhaC subunit with an amino acid sequence similar by about $21-28 \%$ to class I and II PHA synthases, and the PhaE subunit with no similarity at all to class I or II PHA synthases. Class III PHA synthases preferentially accept $(R)$-hydroxyacyl-CoA thioesters with 3 to 5 carbon atoms. Finally, class IV PHA synthases, which catalyze PHA biosynthesis especially in $B$. megaterium, are similar to class III PHA synthases, but PhaE is substituted by the lower molecular PhaR subunit (reviewed by $^{51,52}$ ). 


\section{PHA depolymerases}

Intracellular PHA degradation starts with the catalytic action of PHA depolymerases generating the $(R)$-3-hydroxyalkanoate monomers and oligomers thereof, which are reversibly oxidized by (R)-3-hydroxybutyrate dehydrogenase to acetoacetate, which subsequently undergoes conversion acetoacetyl-CoA by acetoacetyl-CoA synthetase, and, finally, is hydrolyzed by the reversible enzyme 3-ketothiolase to acetyl-CoA. Both intracellular PHA depolymerases, which are needed to maintain the cyclic PHA metabolism in cells, and extracellular PHA depolymerases, which are important for PHA biodegradation, are reported (reviewed by Aneja and Charles).$^{53}$ In a nutshell, intracellular depolymerases are more substrate-specific, while extracellular depolymerases are rather simple esterases, which are also found in mushrooms; they are responsible, e.g., for compostability and biodegradability of PHA. Moreover, intracellular PHA depolymerization always occurs to a certain extent in parallel to PHA polymerization, even under conditions favoring PHA formation, which causes the steady assembly and disassembly of PHA in living cells ("cyclic nature of the PHA metabolism"). ${ }^{54}$ However, under conditions boosting PHA biosynthesis, the activity of intracellular PHA depolymerases is drastically lower than that of PHA synthases. If PHA depolymerases are completely absent, as it is the case in recombinant Escherichia coli harboring PHA synthesis genes, but not the genes encoding PHA depolymerases, the production of ultra-high molecular mass PHA of several MDa can be obtained. ${ }^{55}$ Similar effects were observed after inactivating PHA depolymerase in the genome of Azotobacter vinelandii. ${ }^{56}$ More recently, PHA depolymerases were shown to be specific for oxoester linkages of PHA; thioester bonds in analogue polythioesters (PTEs) are not cleaved by these enzymes. ${ }^{57}$

\section{Short chain length PHA (scl-PHA)}

\section{Typical features of scl-PHA}

In dependence on the type of monomeric building blocks, short chain length PHA (scl-PHA) are differentiated from medium and long chain length PHA ( $m c l$-PHA and $l c l$-PHA). Low glass transition temperatures $\left(T_{g}\right.$ typically between $-40{ }^{\circ} \mathrm{C}$ and $-20{ }^{\circ} \mathrm{C}$ ), a highly amorphous character (degree of crystallinity $X_{c}$ not exceeding $40 \%$ ), wide-ranging melting intervals, low melting temperature typically far below $100{ }^{\circ} \mathrm{C}$, and low degrees of polymerization (molecular mass in most cases below $100 \mathrm{kDa}$ ) differentiate $m c l$-PHA and $l c l$-PHA on the one hand from scl-PHA on the other hand. In the $s c l$-PHA case, high $T_{g}$ around $0{ }^{\circ} \mathrm{C}$ and more, sharp melting points exceeding $180{ }^{\circ} \mathrm{C}$ in the case of highly crystalline PHA, and high molecular masses (up to the MDa range) are typical material features. ${ }^{58}$ Concerning polydispersity indices $\left(P_{i}\right)$ for molecular mass distribution in a PHA sample, generally lower values are reported for $m c l$-PHA than for scl-PHA, hence, scl-PHA display a more heterogeneous distribution of PHA chains of different length in a given sample (reviewed by Zinn). ${ }^{59}$ Fig. 2 illustrates the $s c l$-PHA building blocks discussed in the present review.

Three (3-hydroxypropionate, 3HP), four (3-hydroxybutyrate (3HB), 4-hydroxybutyrate (4HB)), or five (3-hydroxyvalerate (3HV), 4-hydroxyvalerate (4HV), 5-hydroxyvalerate (5HV)) carbon atoms are found in the building blocks of scl-PHA. Regarding their physical characteristics, scl-PHA are similar to classical thermoplasts, therefore, they compete with poly(ethylene) (PE), poly(propylene) (PP), or the bio-based, but chemically polymerized product poly(lactic acid) (PLA); poly(3-hydroxybutyrate) (PHB) is the most commonly occurring and by far the best studied representative of the $s c l$-PHA family. The Gram-negative soil bacterium $C$. necator, for-

Short chain length (scl) PHA monomers

Not branched and chiral:

$\boldsymbol{n}=0, \boldsymbol{R}=\mathrm{H}: \quad$ hydroxyacetate (glycolate) (HA, GA)

$\boldsymbol{n}=0, \boldsymbol{R}=\mathrm{CH}_{3}: \quad$ 2-hydroxypropionate (lactate) (2HP, LA)

$\boldsymbol{n}=1, \boldsymbol{R}=\mathrm{H}: \quad 3$-hydroxypropionate (3HP)

$\boldsymbol{n}=1, \boldsymbol{R}=\mathrm{CH}_{3}: \quad$ 3-hydroxybutyrate (3HB)

$\boldsymbol{n}=1, \boldsymbol{R}=\mathrm{C}_{2} \mathrm{H}_{5}: \quad$ 3-hydroxyvalerate (3HV)

$\boldsymbol{n}=2, \boldsymbol{R}=\mathrm{CH}_{3}: \quad$ 4-hydroxyvalerate (4HV)

Not branched and achiral:

$n=2, R=\mathrm{H}: \quad$ 4-hydroxybutyrate $(4 \mathrm{HB})$

$n=3, R=\mathrm{H}: \quad$ 5-hydroxyvalerate (5HV)

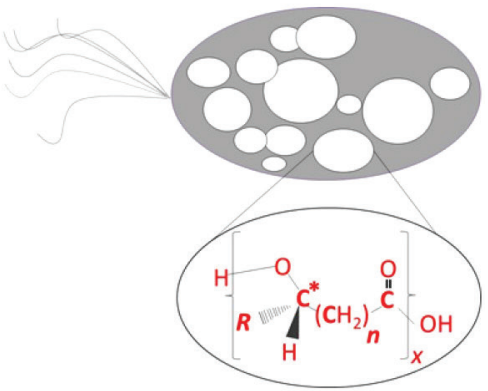

Branched:

$\boldsymbol{n}=0, \boldsymbol{R}=$ isopropyl: 2 -hydroxy-3-methylbutyrate $(2 \mathrm{H} 3 \mathrm{MB})$

Fig. 2 - Monomers of scl-PHA in PHA polyesters discussed in the present review 
merly known as Alcaligenes eutrophus, Ralstonia eutropha, or Wautersia eutropha, ${ }^{60}$ a member of the bacterial family of Burkholderiaceae, is the best investigated scl-PHA production strain with a completely decrypted genome, ${ }^{61}$ which makes this strain intriguing for genetic modification. ${ }^{62}$

\section{4-hydroxybutyrate (4HB) containing scl-PHA}

As an exception among scl-PHA building blocks, 4HB is the only well-studied achiral monomer found in natural PHA. 4HB was first described as PHA constituent by Y. Doi's team, who discovered this novel monomer in PHA samples produced by $C$. necator when being supplied with butyric acid and the 4HB-precursors 4-hydroxybutyric acid or 4-chlorobutyric acid; feeding with butyric acid alone resulted in accumulation of PHB homopolyester. Presence of $4 \mathrm{HB}$ in PHA was confirmed by NMR, and the strong decrease in crystallinity in parallel to increasing $4 \mathrm{HB}$ fraction in PHA was noticed by the authors. Based on these results, this group carried out more in-depth investigations on the properties of this novel class of PHA using $\mathrm{X}$-ray diffraction and DSC studies; they came to the conclusion that the integration of $4 \mathrm{HB}$ building blocks into the highly crystalline PHB matrix causes a considerably stronger drop in lattice crystallinity than shown for comparable fractions of $3 \mathrm{HV}$ in PHBHV copolyesters. ${ }^{63}$ Later, the impact of different $4 \mathrm{HB}$ fractions in poly (3HB-co-4HB) copolyesters on $T_{m}, T_{g}$, and storage modulus $\left(E^{\prime}\right)$ was investigated; it was shown that these parameters decreased with increasing $4 \mathrm{HB}$ fraction in PHA; yield stress and breaking stress only slightly decreased with the increase in the $4 \mathrm{HB}$ contents, while the elongation at break strongly increased. Moreover, improved thermal stability was observed for molded $\mathrm{P}(3 \mathrm{HB}-\mathrm{co}-4 \mathrm{HB})$ copolyester sheets with increased $4 \mathrm{HB}$ fractions. ${ }^{64}$ In addition, it was shown that poly $(3 \mathrm{HB}-\mathrm{co}-4 \mathrm{HB})$ copolyesters are readily biodegradable and biocompatible, with the $4 \mathrm{HB}$ fraction considerably impacting the degradation rate. ${ }^{65}$ Direct comparison between poly(3-hydroxybutyrate-co-3-hydroxyvalerate) (poly $(3 \mathrm{HB}-c o-3 \mathrm{HV})$ ) and poly(3HB-co-4HB) films showed that the latter is faster degraded by random chain scission in phosphate saline buffer than poly (3HB-co-3HV); adding PHB depolymerase to the degradation setups, it was demonstrated that degradation can be accelerated significantly by enzymatic action. Also in these bio-catalyzed experiments, degradation was faster for poly(3HB-co-4HB) films than shown for poly (3HB-co-3HV) or PHB. In all cases, bio-catalyzed degradation was reported to occur via surface erosion. ${ }^{66}$ These experiments opened the door for the broad biomedical application of poly(3HB-co$4 \mathrm{HB})$ and the homopolyester poly $(4 \mathrm{HB})$. Türesin and colleagues resorted to poly (3HB-co-3HV) and poly $(3 \mathrm{HB}-\mathrm{co}-4 \mathrm{HB})$ copolyesters of random distribution to manufacture biodegradable rod-shaped implants; these implants carried Sulperazone ${ }^{\circledR}$ and Duocid $^{\circledR}$, antibiotics applied to treat chronic osteomyelitis. The local in vivo release of these antibiotics by copolyester degradation was studied, showing that the type of antibiotic, drug loading, and additional implant surface coating significantly affect the profile of in vitro drug liberation. For Sulperazone $^{\circledR}$, the rate and period of release from poly (3HB-co-4HB) implants were strongly dependent on the loading with the active ingredient. However, drug dissolution occurred significantly faster than PHA degradation, indicating that the release phenomenon was rather determined by the drug dissolution rate than on PHA degradation or diffusion. When implants were coated with the same type of PHA copolyester, the initial burst effect was significantly reduced, and the liberation rate considerably decreased. Constant drug release from coated implants lasted more than two weeks, while release from uncoated implants did not even take a week. In the case of using Duocid ${ }^{\circledR}$, the generated implants displayed rather smooth surfaces; here, drug release was considerably higher than observed for implants loaded with Sulperazone ${ }^{\circledR}{ }^{67}$

Remarkably, the homopolyester poly $(4 \mathrm{HB})$ has material features completely different from those observed for other scl-PHA, such as PHB or poly $(3 \mathrm{HB}-\mathrm{co}-3 \mathrm{HV})$; it has an outstanding elongation at break of up to $1000 \%$, which makes it highly stretchable and flexible. For other "biopolymers" such as poly(glycolic acid) (PGA), poly(lactic acid) (PLA), or PHB, this value amounts to about 3-6\%, for $\operatorname{poly}(\varepsilon$-caprolactone) (PCL), an elongation at break of about $60 \%$ was reported. Oriented poly $(4 \mathrm{HB})$ fibers have tensile strength values of about $545 \mathrm{MPa}$, which is higher than shown for, e.g., PP sutures (410-460 MPa); this makes such biological fibers interesting as suture material. Moreover, poly(4HB) sutures have a significantly lower Young's modulus than other marketed monofilament sutures. ${ }^{68}$ The company Tepha, Inc., USA, markets a range of PHA-based biomedical products. E.g., poly(4HB)-made TephaFLEX ${ }^{\circledR}$ sutures are US Food and Drug Administration (FDA) approved. By Tepha, Inc., poly(4HB) homopolyester is obtained by a specially engineered cultivation process based on transgenic Escherichia coli K12 as working horse. A range of additional surgical materials based on poly $(4 \mathrm{HB})$ or poly(3HB-co-4HB), e.g., meshes, threads, or films with advantageous mechanical features are produced by Tepha, Inc. (reviewed by ${ }^{69}$ ). Regarding in vivo degradability, poly(4HB) absorption rate is only $8-52$ weeks, which is considerably faster than reported for $\mathrm{PHB}$. 
In the meanwhile, several strain/substrate combinations were reported for biosynthesis of 4HB-harboring PHA. Using wheat straw hydrolysate as main carbon source and $\gamma$-butyrolactone (GBL) as $4 \mathrm{HB}$ precursor, Cesário et al. reported $\mathrm{P}(3 \mathrm{HB}-\mathrm{co}-$ 4HB) biosynthesis by Burkholderia sacchari DSM 17165. These authors also found that, in contrast to GBL, 1,4-butanediol is no suitable 4HB precursor for these organisms, and demonstrated the impact of different glucose/GBL ratios on the PHA fraction in CDM for fed-batch cultivation setups. While cultivation on pure glucose yielded $49.2 \%$ of PHB homopolyester in CDM, only $7.1 \%$ poly $(3 \mathrm{HB}-\mathrm{co}$ 4HB) copolyester (0.046 mol mol-1 4HB in PHA) were obtained on GBL as sole carbon source..$^{70}$ Later, Miranda de Sousa Dias et al. used the same organism in controlled bioreactor cultivations on saccharose from a Brazilian sugar cane mill as main carbon source, with and without co-feeding of GBL. On saccharose only, PHB homopolyester was produced at a volumetric productivity of $1.29 \mathrm{~g} \mathrm{~L}^{-1} \mathrm{~h}^{-1}$, a mass fraction of $0.52 \mathrm{~g}$ PHB per $\mathrm{g}$ biomass, and a final PHB concentration of $36.5 \mathrm{~g} \mathrm{~L}^{-1}$. Co-feeding of GBL resulted in formation of poly (3HB-co-4HB) at a volumetric productivity of $1.87 \mathrm{~g} \mathrm{~L}^{-1} \mathrm{~h}^{-1}$, a mass fraction of $0.72 \mathrm{~g}$ PHA per $\mathrm{g}$ biomass, and a final PHA concentration of $53.7 \mathrm{~g} \mathrm{~L}^{-1}$. Thermoanalysis revealed improved material properties of the copolyester in terms of reduced $T_{m}\left(161{ }^{\circ} \mathrm{C} v s .178\right.$ $187{ }^{\circ} \mathrm{C}$ obtained for the homopolyester PHB) and decreased degree of crystallinity $X_{c}(24 \% v s .71 \%)$, indicating its enhanced suitability for polymer processing. ${ }^{71}$ In another study, Cesário et al. demonstrated the high-cell density production of poly(3HB-co-4HB) by C. necator on crude glycerol and $\mathrm{GBL}$, and of poly (3HB-co-3HV-co-4HB) from crude glycerol, GBL, and the 3HV-related precursor compound propionic acid. ${ }^{72}$ In accordance with previous findings by Lee et al. ${ }^{73}$ propionic acid did not only act as $3 \mathrm{HV}$ precursor, but also boosted the conversion yield of GBL to 4HB. Analogous to other types of scl-PHA, high molecular masses of about $1 \mathrm{MDa}$ were obtained for the produced biopolyesters. ${ }^{72}$ Similar attempts were accomplished by Hermann-Krauss et al. using the haloarchaeon Haloferax mediterranei to produce poly(3HB-co$4 \mathrm{HB}$ ) from CGP, a side-product of biodiesel production, plus GBL as $4 \mathrm{HB}$ precursor; in this case, the terpolyester poly(3HB-co-3HV-co-4HB) was produced. ${ }^{74}$ This surprising outcome is based on a metabolic particularity of this organism, namely, $3 \mathrm{HV}$ production from structurally unrelated carbon sources such as sugars or glycerol. Additional poly(3HB-co-3HV-co-4HB) terpolyesters were produced by Aziz and colleagues, who cultivated $\mathrm{Cu}$ priavidus sp. USMAA2-4, an organism isolated from a Malaysian lake, in a high-productive DO- stat fed-batch process in a $2 \mathrm{~L}$ bioreactor on oleic acid, pentanol (3HV precursor), and GBL. Based on the substrate mix, fractions of $3 \mathrm{HB}, 3 \mathrm{HV}$, and $4 \mathrm{HB}$ in PHA varied between 62 and $86 \%$ (3HB), 9 and $13 \%(3 \mathrm{HV})$, and 4-24\% (4HB), respectively. Similar to previous studies, presence of $3 \mathrm{HV}$ and $4 \mathrm{HB}$ considerably reduced the crystallinity of the produced PHA samples, thus enhancing the physical and thermomechanical polyester properties, which was especially evident by lower melting points and improved elongation at break. Moreover, it was revealed that the tensile strength of the terpolyesters increases with increasing $M_{w}{ }^{75}$

In recent time, strategies have been developed to produce poly(3HB-co-4HB) in an inexpensive and efficient way. In this context, the osmophilic halobacterium Halomonas bluephagenesis TD01, an organism naturally not producing 4HB-containing PHA, was genetically engineered by inserting the gene orfZ, which encodes Clostridium kluyveri 4HB-CoA transferase, making this strain capable of poly $(3 \mathrm{HB}-\mathrm{co}-4 \mathrm{HB})$ production when cultured on glucose and the $4 \mathrm{HB}$ precursor GBL. Pilot scale cultivations in $1-\mathrm{m}^{3}$ bioreactors resulted in $83 \mathrm{~g} \mathrm{~L}^{-1}$ CDM, $0.61 \mathrm{~g}$ poly(3HB-co-4HB) per g CDM, and 16 mol-\% 4HB in the copolyester; remarkably, it was possible to carry out these cultivations under open, non-sterile conditions due to the high salinity of the cultivation medium $\left(60 \mathrm{~g} \mathrm{~L}^{-1} \mathrm{NaCl}\right) .^{76}$ Janwen et al. accomplished further engineering of this strain to make it capable of producing poly $(3 \mathrm{HB}-\mathrm{co}-4 \mathrm{HB})$ from glucose as sole carbon source; here, comparative genome analysis was applied for "pathway debugging", resulting in $25 \mathrm{~mol}-\%$ 4HB in the copolyester. ${ }^{77}$ These activities were followed by further studies, which demonstrated the viability of using the wild type form of this strain on a larger scale (5 $\mathrm{m}^{3}$ ) for PHB production under continuous and non-sterile conditions, using waste gluconate as inexpensive substrate. ${ }^{78}$ Recent development with this auspicious microorganism involved further genetic engineering via genome-wide random mutagenesis in order to make the strain resistant to toxic metabolites such as ethanol or short carboxylic acids during high cell density cultivation. Further genetic engineering of obtained strains was carried out for overexpression of PHA synthesis genes, resulting in $90 \mathrm{~g} \mathrm{~L}^{-1} \mathrm{CDM}$ and 0.79 g PHA per g CDM in $7 \mathrm{~L}$ bioreactor setups, which is considerably higher than values obtained with the wild type strain $\left(81 \mathrm{~g} \mathrm{~L}^{-1}\right.$ and $0.97 \mathrm{~g} \mathrm{~g}^{-1}$, respectively). ${ }^{79}$ Other representatives of Halomonas sp. are currently being investigated for production of PHB homopolyester under non-sterile conditions, such as Halomonas halophi$l a$, a strain which converts various inexpensive carbon sources, such as lignocellulose hydrolysates, molasses, or hydrolyzed cheese whey, for biomass 
growth and PHB formation; up to $0.8 \mathrm{~g}$ PHB per $\mathrm{g}$ CDM were obtained under halophilic cultivation conditions $\left(66 \mathrm{~g} \mathrm{~L}^{-1} \mathrm{NaCl}\right)$. Remarkably, the authors demonstrated that molecular mass of the produced PHB strongly depends on the salinity of the cultivation medium; higher salinity (up to $100 \mathrm{~g} \mathrm{~L}^{-1}$ ) increases $M_{w}$ to up to $810 \mathrm{kDa}$, which is considerably higher than about $420 \mathrm{kDa}$ obtained under moderately halophilic conditions $\left(20 \mathrm{~g} \mathrm{~L}^{-1} \mathrm{NaCl}\right)$. At the same time, polydispersity of the polymer by trend increases with increasing salinity. This finding allows fine-tuning molecular mass by triggering the salt concentration. ${ }^{44}$

As shown by Lv and colleagues, clustered regularly interspaced short palindromic repeats interference (CRISPRi) can be used to trigger poly(3HBco-4HB) biosynthesis in $E$. coli by parallel fine-tuning gene expression and regulating the expression of multiple genes. For this purpose, a pathway was constructed in E. coli for poly(3HB-co$4 \mathrm{HB})$ biosynthesis from glucose. The native gene sad, which encodes succinate semi-aldehyde dehydrogenase in $E$. coli, was expressed under the control of CRISPR $i$ using five specially designed single guide RNAs (sgRNAs) to regulate the carbon flux to $4 \mathrm{HB}$ biosynthesis; poly (3HB-co-4HB) with 1-9 mol- $\%$ 4HB were obtained with this system. Moreover, succinate, generated by succinyl-CoA synthetase and succinate dehydrogenase, respectively, (encoded by genes $s u c C, s u c D$ and $s d h A, s d h B$ ) was directed favorably towards the $4 \mathrm{HB}$ precursor by applying selected sgRNAs (sucC2, sucD2, sdhB2 and $s d h A 1$ ) by CRISPRi. Depending on the expression levels of down-regulated gene, the resulting molar 4HB fraction in copolyesters ranged from 1.4 to $18.4 \mathrm{~mol}-\%{ }^{80}$

\section{Sulfur-containing scl-PHA analogues}

Apart from naturally occurring scl-PHA, which constitute polyoxoesters of alkanoic acids, biosynthesis of polythioesters (PTEs), polymers of non-naturally-occurring mercaptoalkanoic acids, such as poly(3-mercaptopropionate), also find attention in the relevant scientific literature. ${ }^{81}$ These materials are biosynthesized by the action of PHA synthases in recombinant prokaryotes (Cupriavidus sp., E. coli, and Advenella sp.) starting from organosulfur compounds, namely, mercaptoalkanoic acids, with 3-mercaptopropionate (3MP) being the best described among suchprecursors. ${ }^{82}$ As a major shortcoming, the preparation of these mercaptoalkanoic acids is exceedingly expensive, which currently hampers an economically feasible production of these compounds on the industrial scale. However, PTEs revealed lower crystallinity and, for some cases, higher thermal stability in comparison to their corresponding oxoester analogues (PHAs), which makes them stimulating for different technological applications. In addition, PTEs display astonishing persistence against biodegradation; no biodegradation of PTEs was observed when using a variety of bacteria and fungi capable of degrading PHA polyoxoesters. ${ }^{83}$ To date, no organisms or habitats have been detected were PTE becomes biodegraded. ${ }^{82}$ Remarkably, copolyesters of $3 \mathrm{HB}$ and $3 \mathrm{MP}$, poly(3HB-co-3MP), are readily degraded by natural depolymerases due to their specificity for the oxoester bonds. ${ }^{57}$ When aspiring a cost-efficient production of persistent organosulfur polymers, it would be indispensable to develop metabolic pathways allowing for de novo biosynthesis of the sulfur-containing compounds. ${ }^{82}$

\section{Medium chain length PHA (mcl-PHA)}

\section{Typical features of mcl-PHA}

Mcl-PHA, typically containing monomers with 6 to 14 carbon atoms, are considerably less crystalline than $s c l$-PHA. Some $m c l$-PHA contain building blocks with chemical groups, e.g., olefinic or epoxy-groups, which allow for their chemical or enzymatic post-synthetic modification in order to finetune the polymer properties. Macroscopically, $m c l$-PHA often have the appearance of biological rubbers or latexes; due to their remarkably low $T_{g}$ values, their amorphous structure does not easily change into a crystalline structure, which prevents them from becoming brittle even at temperatures well below freezing. Pseudomonas putida (formerly known as Pseudomonas oleovorans) is the mclPHA production strain most frequently used by research groups all over the world. ${ }^{59}$ This organism has the beneficial feature of incorporating functionalized (unsaturated, halogenated, epoxy-group harboring, etc.) monomers in growing $\mathrm{mcl}$-PHA chains when supplied with suitable functionalized precursor compounds. In this context, a typical rubber-like material was produced already in 1993 by de Koning and colleagues, who generated a mcl-PHA consisting of saturated and unsaturated building blocks by co-feeding Pseudomonas oleovorans with mixtures of n-octane and 1-octene. From the praxis-oriented perspective, the obtained material displayed inappropriate material features in terms of too low melting point and insufficient crystallization rate; however, the authors were able to profit from the functional (olefinic) groups, and subjected the polyester to post-synthetic modification by crosslinking the polyester chains via their pendant unsaturated groups with electron-beam irradiation. The final material revealed constant properties over a temperature range from $-20^{\circ} \mathrm{C}$ to $170{ }^{\circ} \mathrm{C}$, and was still biodegradable. ${ }^{84}$ Regarding the physical properties, $m c l$-PHA typically show expedient elongation at 


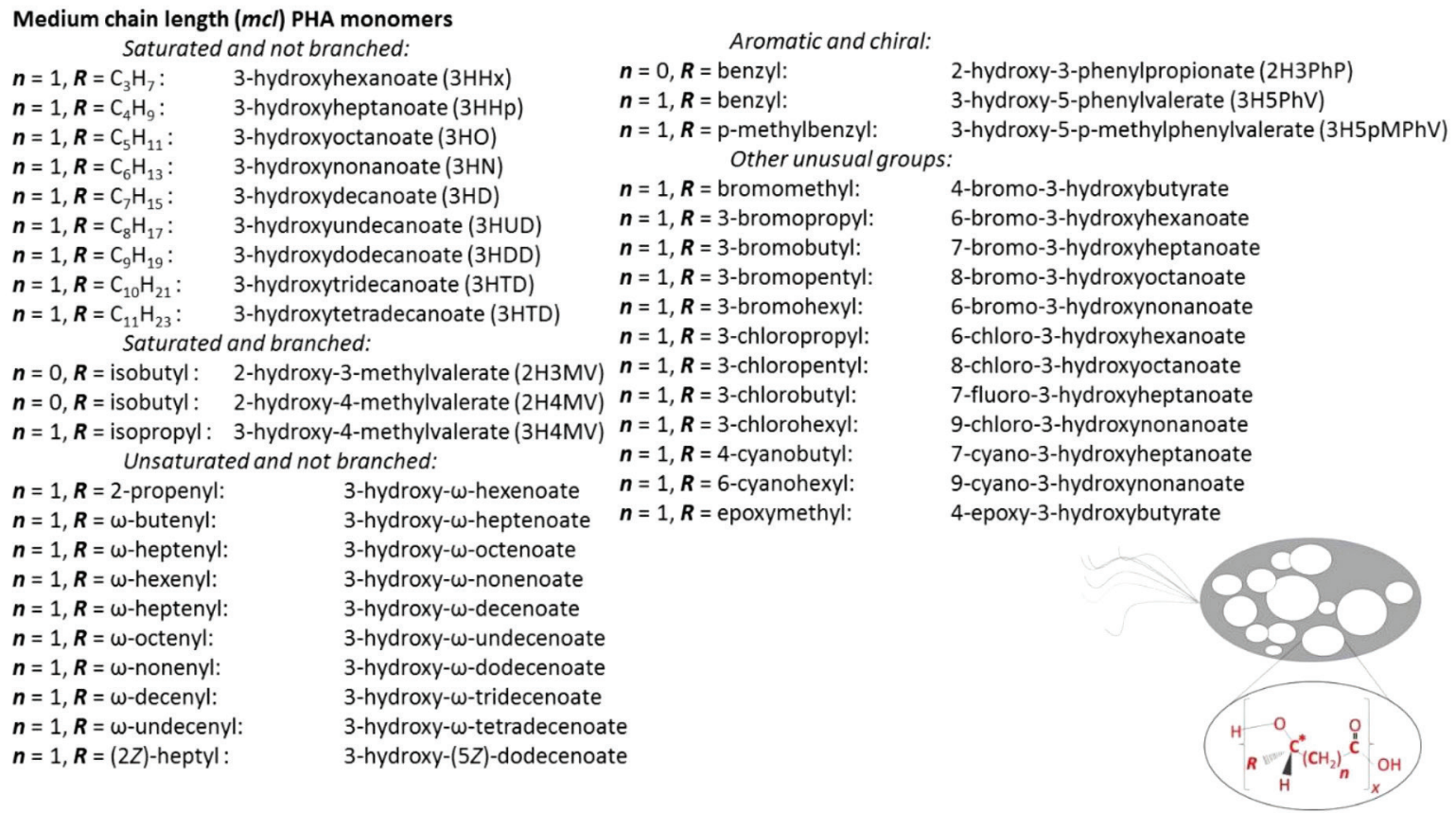

Fig. 3 - Monomers of mcl-PHA in PHA polyesters discussed in the present review

break of some $100 \%$; for the scl-PHA PHB, this value is only about $6 \%$. Comparing values for tensile strength of different types of PHA, most $s c l$ PHA representatives have values of about $30-40$ $\mathrm{MPa}$, which is drastically higher than the value of about $10 \mathrm{MPa}$, which is usually measured for $\mathrm{mcl}$ PHA. In this context, the scl-PHA homopolyester poly(4HB) constitutes a remarkable exception, having an elongation to break of about $1000 \%$; moreover, the $T_{g}$ of $\mathrm{P}(4 \mathrm{HB})\left(\right.$ about $-50{ }^{\circ} \mathrm{C}$ ) is in the same range as for $m c l$-PHA. ${ }^{58}$ Regarding molecular mass, $m c l$-PHA produced by class II PHA synthases have typically lower molecular weights $(50-500 \mathrm{kDa})$ than scl-PHA produced by the enzymatic action of class I synthase. ${ }^{85}$ As an example, Rodrigues and colleagues recently reported the production of mcl-PHA copolyesters mainly consisting of 3-hydroxyhexanoate (3HHx) and 3-hydroxy-9-octadecenoate when cultivating the strains $C$. necator (IPT 026 and IPT 027) and Burkholderia cepacia (IPT 119 and IPT 400) on crude palm oil. These polymers, highly amorphous and thermostable materials, revealed molecular masses typically low for $m c l$-PHA $(170-400 \mathrm{kDa}){ }^{86}$ Fig. 3 illustrates the $m c l$-PHA building blocks discussed in the present review.

\section{Mcl-PHA biosynthesis via $\beta$-oxidation of fatty acids}

Typically, $m c l$-PHA is produced by microbes via $\beta$-oxidation of fatty acids towards mixtures of $(S)$-acyl-CoAs of different length, which, after enzymatic isomerization to the corresponding $(R)$ acyl-CoAs, are typically polymerized towards struc- turally related $\mathrm{mcl}$-PHA copolyesters by class II synthases. ${ }^{87}$ Therefore, mcl-PHA generally constitute heteropolyesters of different building blocks with six or more carbon atoms. As an example, the strains Pseudomonas citronellolis and Pseudomonas chlororaphis accumulated $m c l$-PHA of typical sticky, resin-like character, consisting of $3 \mathrm{HHx}$, 3-hydroxyheptanoate (3HHp), 3-hydroxyoctanoate (3HO), 3-hydroxynonanote $(3 \mathrm{HN})$, and 3-hydroxydecanoate (3HD), when cultivated on fatty acids methyl esters in fed-batch bioreactor setups. ${ }^{88,89}$ In 2007, Liu and Chen demonstrated that $\beta$-oxidation weakened mutants of $P$. putida KT2442, which were generated by knocking out 3-ketoacyl-CoA thiolase and 3-hydroxyacyl-CoA dehydrogenase, when cultivated on tetradecanoic acid as sole carbon source, produce mcl-PHA with high shares (up to $49 \%$ ) of 3-hydroxytetradecanoate (3HTD), and low shares (3\%) of $3 \mathrm{HHx}$ if compared to the wild type strain. Remarkably, at the same time, the authors found oligomers of 3HTD in the cells, which evidences the restricted potential of the strain of polymerizing 3HAs of such long chain length. Moreover, increased shares of 3HTD resulted in higher crystallinity and tensile strength than reported for typical $m c l$-PHA with building blocks not longer than 3-hydroxydodecanoate (3HDD). ${ }^{90}$

Mcl-PHA biosynthesis via fatty acid de novo biosynthesis

In addition, $m c l$-PHA production starting from fatty acid de novo synthesis from unrelated carbon sources, such as sugars or glycerol, was described. ${ }^{91}$ 
In contrast to $\beta$-oxidation, fatty acid de novo synthesis directly generated the $(R)$-isomers of acylCoAs. In this context, Sathiyanarayanan and colleagues only recently reported the production of poly(3HTD-co-3HDD-co-3HD-co-3HO) by the psychrophilic bacterium Pseudomonas sp. PAMC 28620, isolated from Arctic glacier fore-field soil. Using glycerol as sole carbon source, high PHA fractions of more than 50 wt.\% in CDM were achieved. Such exotic $m c l$-PHA copolyesters were also obtained by exchanging glycerol with carbohydrates, which revealed low crystallinity and high thermal stability; moreover, as a typical feature also for other $m c l$-PHA, these materials had a rather low molecular mass of only about $30 \mathrm{kDa} .{ }^{92}$ Similar findings were reported by Muangwong et al., who investigated four strains isolated in Thailand from soils contaminated with used cooking oil (UCO). These strains, classified as Acinetobacter sp., Pseudomonas sp., Enterobacter sp., and Bacillus sp., accumulated $\mathrm{mcl}$-PHA copolyesters consisting of $3 \mathrm{HO}$ and the unsaturated building block 3-hydroxy-(5Z)-dodecenoate from crude glycerol stemming from UCO-based biodiesel production. Similar to the results reported by Sathiyanarayanan et al., molecular mass amounted to about 30-40 kDa. Surprisingly, homopolyester poly(3-hydroxy-(5Z)dodecenoate) was accumulated by a Bacillus sp. when the cultivation was prolonged to $96 \mathrm{~h} .{ }^{93} \mathrm{Ge}-$ netic engineering was used by Mendonça et al., who equipped a PHA-negative mutant of $B$. saccha$r i$ with Aeromonas sp. PHA biosynthesis genes. This genetic construct was able to accumulate copolyesters of $s c l-3 \mathrm{HA}(3 \mathrm{HB})$ and $m c l-3 \mathrm{HA}$ from sucrose as sole carbon source. ${ }^{94}$

\section{Dual nutrient limited cultivation to produce mcl-PHA of tailored composition}

Mcl-PHA biosynthesis is often based on the use of growth-inhibiting substrates like fatty acids, which drastically restricts volumetric productivity of the process; to overcome the inhibiting effect, one can resort to chemostat cultivations under a dual nutrient limited growth (DNL) regime, where both carbon and nitrogen sources are supplied continuously at concentrations immediately metabolized by the cells. The DNL concept originates from the biological law that concentration and composition of biomass and growth rate are not determined by a single limiting factor, but by the synergistic action of at least two limiting nutrients (reviewed by Zinn et $a l . .^{95}$ ). Substrate toxicity depends on the substrate's concentration, hence, biomass growth is typically not influenced when the substrate is instantaneously converted and does not surpass a given threshold concentration. In the context of $\mathrm{mcl}$ PHA production, DNL cultivation was tested using continuous cultivation of $P$. putida GPo1 on mixed carbon sources; here, $m c l$-PHA of unprecedented monomeric composition were produced. Mixtures of 5-phenylvalerate, octanoate, and 10-undecenoate yielded diverse poly(3-hydroxy-5-phenylvalerateco-3-hydroxyalkanoate-co-3-hydroxy- $\omega$-alkenoate) copolyesters composed of aromatic, saturated, and unsaturated monomers. Depending on the substrate mix, the aromatic (3-hydroxy-5-phenylvalerate, $3 \mathrm{H} 5 \mathrm{PhV}$ ) fraction ranged from 0 to $0.52 \mathrm{~mol} \mathrm{~mol}^{-1}$; substrate feeding was accomplished at a rate according to substrate conversion by the microbes, which kept the actual substrate concentrations at almost zero. Surprisingly, increasing aromatic fraction correlated with a linear increase in the $T_{g}$ from $-37.6{ }^{\circ} \mathrm{C}$ to $-6{ }^{\circ} \mathrm{C} .{ }^{96}$ Further DNL cultivation was carried out using mixtures of lactic acid and the aromatic compounds p-methylphenylvaleric acid or phenylvaleric acid, respectively, as co-substrates. When using p-methylphenylvaleric acid as co-substrate, the mcl-PHA homopolyester poly(3-hydroxy-5-p-methylphenylvalerate) was produced, a material displaying properties different to other, often completely amorphous, $m c l$-PHA copolyesters: it revealed significant crystallinity and a sharp $T_{m}$ at $99{ }^{\circ} \mathrm{C}$, which is in contrast to other described $\mathrm{mcl}$ PHA samples. Using phenylvaleric acid as co-substrate, the homopolyester poly $(3 \mathrm{H} 5 \mathrm{PhV})$ was produced, which displayed typical mcl-PHA material properties. It should be emphasized that chemostat cultivation processes, especially using DNL substrate supply, might be the only efficient strategy to thrive PHA producing strains on toxic substrates like the described aromatic compounds. ${ }^{97}$

\section{Long chain length PHA (Icl-PHA)}

Lcl-PHA are still scarcely described; these polyesters contain building blocks with more than 14 carbon atoms. The first report on a PHA containing 3-hydroxypentadecanoate (3HPD) was delivered by Barbuzzi and colleagues, who found $2 \%$ 3HPD in PHA accumulated by Pseudomonas aeruginosa ATCC 27853 when cultivated on pentadecanoic acid as sole carbon source. ${ }^{98}$ A recent report describes $l c l$-PHA production by $C$. necator IPT027 from crude palm oil as inexpensive carbon source, which contains a high fraction of unsaturated fatty acids. This polymer consisted predominately of $3 \mathrm{HD}$ and diverse saturated and unsaturated building blocks with 18 carbon atoms, namely 3-hydroxyoctadecanoate, 3-hydroxy-9-octadecenoate, and 3-hydroxy-9,12-octadecadienaote. The material was of highly amorphous character and revealed low polydispersity and high thermal stability. In the same study, it was demonstrated that, switching from $C$. necator IPT027 to Burkholderia cepacia IPT400 while using the same substrate and cultiva- 


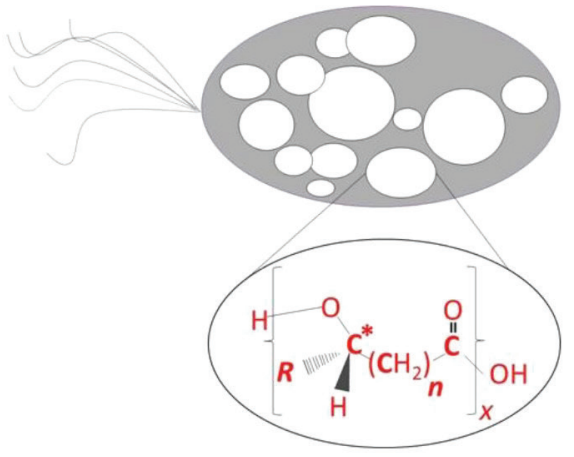

Long chain length (ICl) PHA monomers

Saturated:

$\boldsymbol{n}=1, \boldsymbol{R}=\mathrm{C}_{12} \mathrm{H}_{25}:$ 3-hydroxypentadecanoate (3HPD)

$\boldsymbol{n}=1, \boldsymbol{R}=\mathrm{C}_{13} \mathrm{H}_{27}:$ 3-hydroxyhexadecanoate (3HHxD)

$\boldsymbol{n}=1, \boldsymbol{R}=\mathrm{C}_{14} \mathrm{H}_{29}: \quad$ 3-hydroxyheptadecanoate (3H HpD)

$\boldsymbol{n}=1, \boldsymbol{R}=\mathrm{C}_{15} \mathrm{H}_{31}: \quad$ 3-hydroxyoctadecanoate (3HOD)

Unsaturated:

$\boldsymbol{n}=1, \boldsymbol{R}=\mathrm{C}_{15} \mathrm{H}_{23}:$ 3-hydroxy-9-octadecenoate

$\boldsymbol{n}=1, \boldsymbol{R}=\mathrm{C}_{15} \mathrm{H}_{21}$ : 3-hydroxy-9,12-octadecadienaote

Fig. 4 -Monomers of mcl-PHA in PHA polyesters discussed in the present review

tion conditions, the polymer structure arrangement changed considerably, as evidenced by a decrease in crystallinity from $X_{c}=48.47 \%$ to $X_{c}=27.80 \%$, which might have been due to the higher fractions of unsaturated building blocks found in the PHA produced by $B$. cepacia IPT400 than by $C$. necator IPT027. ${ }^{99}$ An even longer building block was detected only recently by Impallomeni et al. ${ }^{100}$ These authors cultivated $P$. aeruginosa ATCC 27853 on long odd-numbered fatty acids with 17 to 21 carbon atoms (heptadecanoic acid, nonadecanoic acid, and heneicosanoic acid) under nitrogen-limited conditions. The resulting polyesters contained odd-numbered $3 \mathrm{HAs}$ from $3 \mathrm{HV}$ to the previously unprecedented monomer 3-hydroxyheptadecanoate (3HHpD). The materials were reported as sticky, soft, rubber-like materials having $T_{g}$ values between -45 and $-39{ }^{\circ} \mathrm{C}, T_{m}$ values between 48 and $52{ }^{\circ} \mathrm{C}$, melting enthalpies of about $11 \mathrm{~J} \mathrm{~g} \mathrm{~g}^{-1}$, and low molar masses between 77 and $188 \mathrm{~kg} \mathrm{~mol}^{-1}$. This study also showed, for the first time, that presence of magnesia ions in the cultivation medium is needed to allow the metabolization of such long-chain fatty acids towards PHA. ${ }^{100}$ The longest PHA building block detected to date in natural PHA, 3-hydroxyoctadecanoate (3HOD), was described by Ray and Kalia, who obtained PHA copolyesters consisting of 3HD, 3-hydroxyhexadecanoate, and 3HOD after co-feeding Bacillus thuringiensis EGU45 with glucose, CGP, and propionic acid. ${ }^{101}$ The same building block was also detected by Guo and colleagues in PHA samples produced by a genetically engineered Pseudomonas mendocina cultivated on glucose as sole carbon source. ${ }^{102}$ Fig. 4 illustrates the $l c l$-PHA building blocks discussed in the present review.

\section{PHA homo- and heteropolyesters}

\section{$3 H V$-containing heteropolyesters}

The most frequently described member of the PHA family is PHB, the homopolyester of $3 \mathrm{HB}$. This material was discovered about 90 years ago by Maurice Lemoigne (reviewed by ${ }^{103}$ ), and, until
1974, it was considered the only type of natural PHA existing in microbes. Due to its high brittleness, high melting point (about $180{ }^{\circ} \mathrm{C}$ ), and high crystallinity (typically 60-70\%), the application of PHB homopolyester, especially for packaging purposes, was strongly limited. ${ }^{104}$ In 1974 , Wallen and Rohwedder isolated microbes from sewage water; the authors remarked that properties of the PHA produced by this microbial community differed considerably from those of PHB homopolyester; significantly lower $T_{m}$ below $100{ }^{\circ} \mathrm{C}$ and solubility in cold ethanol surprised these authors. Based on GC-MS analyses, it was confirmed that the polymer contained, beside $3 \mathrm{HB}$, also $3 \mathrm{HV}$ units. To a minor extent, even $3 \mathrm{HHx}$ was discovered as the first unambiguously identified $m c l$-PHA building block; moreover, presence of $3 \mathrm{HHp}$ was evidenced, but not confirmed. ${ }^{105}$ The number of PHA monomers grew further in 1983, when Findley and White reported the composition of PHA extracted from estuarine sediments; these biopolyesters consisted of at least 11 different monomeric building blocks, among them, $3 \mathrm{HB}, 3 \mathrm{HV}, 3 \mathrm{HHx}, 3 \mathrm{HHp}$, and $3 \mathrm{HO}$ were unambiguously identified by GC-MS; evidence was also given for iso-branched building blocks with five and seven carbon atoms, moreover, 3 peaks in the chromatogram were not assigned to a defined structure of a monomer. Parallel investigation described in this study revealed the complex composition of a PHA extracted from Bacillus megaterium ssp. globiguii, an organism containing Class IV synthase, when cultivated on a nitrogen-free minimal medium; surprisingly, this polymer turned out to contain, beside 3HB (95\%), also $3 \mathrm{HHp}(3 \%)$, traces of $3 \mathrm{HV}$ and an additional, yet unidentified building block. ${ }^{106}$ This was in contrast to other $B$. megaterium ssp. isolated later, such as $B$. megaterium uyuni isolated from a Bolivian salt lake, which accumulated PHB homopolyester when grown on glucose-based, nitrogen-free minimal medium. ${ }^{45}$ Soon after, it was shown that the best-described scl-PHA production strains, like $C$. necator, require supplementation of precursor compounds 
structurally related to $3 \mathrm{HV}$, if the production of poly (3HB-co-3HV) copolyesters instead of brittle PHB homopolyester is aspired; this was demonstrated for the first time by co-feeding butyric acid and the $3 \mathrm{HV}$ precursor pentanoic (=valeric) acid to A. eutrophus (today: C. necator); using pentanoic (=valeric) acid as sole carbon source, the molar $3 \mathrm{HV}$ fraction in PHA reached up to $90 \%$; based on the use of ${ }^{13} \mathrm{C}$-labeled substrates, the authors elucidated the pathway for poly(3HB-co-3HV) copolyester biosynthesis by this strain, namely the conversion towards valeryl-CoA, the subsequent $\beta$-oxidation to 3-hydroxyvaleryl-CoA, and direct incorporation of the latter into PHA. ${ }^{107}$ Later, propionic acid was studied as another $3 \mathrm{HV}$-precursor compound for $C$. necator; this compound is intracellularly converted to propionly-CoA, which couples with acetyl-CoA, thus forming the oxoacyl-thioester 3-keto-valeryl-CoA, which, after reduction to 3-hydroxyvalery-CoA, is incorporated as $3 \mathrm{HV}$ into growing PHA chains by class I PHA synthase (reviewed by Braunegg et al. ${ }^{50}$ ). It was demonstrated by Lefebvre et al. that increased $3 \mathrm{HV}$ fractions in PHA can be achieved when restricting the dissolved oxygen concentration during cultivations supplied with glucose and sodium propanoate; this strategy prevents the oxidative decarboxylation of 3-hydroxyvalery-CoA towards 3-hydroxybutyryl-CoA, which generates $3 \mathrm{HB}$ at the expense of $3 \mathrm{HV}$. However, this restricted oxygen supply results in reduced overall PHA productivity. ${ }^{108}$ Using mixed microbial cultures supplied with volatile fatty acids, Montano-Herrera and colleagues noticed that the fraction of $3 \mathrm{HV}$ in PHA strongly depended on the instantaneous availability of reducing equivalents NAD $(\mathrm{P})$ $\mathrm{H} .{ }^{109}$ In the meanwhile, poly (3HB-co-3HV) production is described for a range of other different strains, such as Burkholderia parafungorum (previously known as Pseudomonas hydrogenovora), ${ }^{110}$ Hydrogenophaga pseudoflava, ${ }^{110}$ etc. In addition to propionic acid and valeric acid, inexpensive $3 \mathrm{HV}$ precursors are currently under investigation in order to save material costs for the bioprocess; in this context, levulinic acid, a material easily accessible from conversion of lignocellulosic biomass, was successfully used in its pure form, ${ }^{111,112}$ or as constituent of acid hydrolyzed spruce wood $^{113}$ for poly(3HB-co-3HV) production. More recently, waste lipids were ozonolytically hydrolyzed towards a cocktail containing a certain amount of compounds with an odd number of carbon atoms, which in turn acted as $3 \mathrm{HV}$ precursor for $C$. necator. ${ }^{114}$ Kumar and colleagues reported an intriguing study on the use of microbial hydrolyzed agro-food waste for poly (3HB-co-3HV) production by different Gram-positive production strains. Among the investigated strains, Bacillus thuringensis showed expedient potential for PHA accumulation, with composition of the biopolyesters differing depending on the applied agro-food waste; while application of hydrolyzed pea shells generated PHB homopolyester, poly (3HB-co-3HV) production was achieved using hydrolyzed apple pomace, onion peels or potato peals. ${ }^{115} \mathrm{~A}$ different direction is possible when using representatives of the haloarchaea, a group of ancient organisms occupying highly saline habitats. As demonstrated in the case of $H f x$. mediterranei and Halogeometricum borinquense, such organisms produce poly(3HB-co-3HV) without the need for $3 \mathrm{HV}$-related precursor compounds; feeding these strains with simple pure sugars, ${ }^{116}$ hydrolyzed bagasse, ${ }^{117}$ or glycerol ${ }^{74}$ is sufficient to make them produce copolyesters. Only recently, copolyester production from inexpensive unrelated substrates was detected also in eubacteria, when Bacillus licheniformis PL26 was shown to accumulate poly(3HB-co-3HV) from crude glycerol from the biodiesel production. ${ }^{118}$

\section{Nodax-copolyesters}

Although the incorporation of $3 \mathrm{HV}$ into the crystalline PHB matrix can reduce $X_{c}$ and $T_{m}$ of resulting poly(3HB-co-3HV) copolyesters, this effect is not sufficient for many applications due to the so-called "isodimorphism", describing the phenomenon of $3 \mathrm{HV}$ units being well integrated into the $3 \mathrm{HB}$ crystal lattice and vice versa, which prevents expedient disruption of crystallinity. Higher lattice disruption is obtained when integrating $\mathrm{mcl}$-PHA building blocks, such as $3 \mathrm{HHx}$ or longer analogues. Resulting poly(3HB-co-mcl-3HA) with a rather low fraction of $m c l$-HA copolyesters are termed "Nodax"-PHA. These polymers are accessible either via bacterial fermentation of the wild type bacteria Aeromonas hydrophila or Aeromonas caviae, hence, organisms containing class III PHA synthases, on long chain fatty acids, or by genetically engineered $C$. necator harboring both the natural class I PHA synthase and Pseudomonas fluorescens GK13 class II PHA synthase on sugars and fatty acids. Poly(3HB-co-3HHx) is the most simple type of "Nodax"-PHA. Based on the decreased crystallinity, Nodax copolymers are characterized by high toughness and ductility, and show convenient thermal properties similar to those of PE. Incorporation of $m c l$-3HA monomers efficiently lowers the $X_{c}$ and $T_{m}$ similar as observed for $\alpha$-olefins (1-alkenes) in linear LDPE, resulting in a broad window of processibility, hence, a large difference between $T_{m}$ and decomposition temperature $T_{d}$, which facilitates a range of processing techniques. While the chain length of the mcl-3HA component has only insignificant effect on reduction of $T_{m}$ and $X_{c}$, it strongly dictates the polymers flexibility. Nodax copolyes- 
ters are reported to be completely biodegradable in both aerobic and anaerobic environments, are compatible with other (bio)polymers such as PLA, and exert expedient oxygen and flavor barrier properties, which makes them auspicious for packaging application in the food sector (reviewed by Noda et $\left.a l .{ }^{119}\right)$.

\section{3-hydroxypropionate (3HP) containing scl-PHA hetero- and homopolyesters}

In 1991, Nakamura and colleagues from Y. Doi's research team discovered 3HP as a new PHA building block when supplying nitrogen-depriviated C. necator cultures with 3-hydroxypropionate (3HP), 1,5-pentanediol, or 1,7-heptanediol as sole carbon sources. Depending on the precursor, 3HP fractions in poly (3HB-co-3HP) copolyesters varied between 0 and 7 mol- $\%$, with increasing $3 \mathrm{HP}$ fractions resulting in a decrease in $T_{m}$ from $178{ }^{\circ} \mathrm{C}$ (PHB homopolyester) to $150{ }^{\circ} \mathrm{C}$ for poly (3HB-co-7 $\%-3 \mathrm{HP}) .{ }^{120}$ Based on the fact that $3 \mathrm{HP}$ turned out as the precursor resulting in highest $3 \mathrm{HP}$ fractions, the authors carried out follow up studies by co-feeding Alcaligenes latus ATCC 29713 with sucrose and $3 \mathrm{HP}$ at different ratios in $48 \mathrm{~h}$ shaking flask cultivations. On sucrose only, PHB homopolyester was accumulated, whereas 50/50 mixtures of sucrose/precursor resulted in highest 3HP molar fractions of 26 $\%$ in poly (3HB-co-3HP). However, CDM dropped from $4 \mathrm{~g} \mathrm{~L}^{-1}$ (pure sucrose; $0.6 \mathrm{~g} \mathrm{~g}^{-1} \mathrm{PHA}$ in $\mathrm{CDM}$ ) to $2 \mathrm{~g} \mathrm{~L}^{-1}$ (50/50 mixtures; $0.29 \mathrm{~g} \mathrm{~g}^{-1}$ PHA in CDM); using $3 \mathrm{HP}$ as sole carbon source, bacterial growth was completely inhibited, which indicated the inability of $A$. latus to convert $3 \mathrm{HP}$ to acetyl-CoA, which is in contrast to the above report using $C$. necator, which is able to grow on $3 \mathrm{HP}$ as sole carbon source. With increasing 3HP fractions, $T$ dropped from $177{ }^{\circ} \mathrm{C}(\mathrm{PHB})$ to $85^{\circ} \mathrm{C}$ (poly $(3 \mathrm{HB}-$ co-26\%-3HP)); at the same time, $T_{\text {a }}$ also decreased from $4{ }^{\circ} \mathrm{C}(\mathrm{PHB})$ to $-8{ }^{\circ} \mathrm{C}$ (poly $\left(3 \mathrm{HB}^{g}-\mathrm{co}-26 \%-3 \mathrm{HP}\right)$. Together with the trend of heat fusion enthalpy values $\left(\delta H_{m}\right)$ also decreasing with increasing 3HP fraction, the authors assumed that 3HP lowers the crystallinity of copolyesters containing this monomer. ${ }^{121}$ This was confirmed in subsequent studies by Shimamura and colleagues, who observed decreasing crystallinity in $A$. latus poly (3HB-co-3HP) with up to $0.88 \mathrm{~mol} \mathrm{~mol}^{-1} 3 \mathrm{HP}$ using $\mathrm{X}$-ray diffraction characterization; this study also revealed improved enzyme-catalyzed biodegradability of poly(3HB-co3HP) films containing increased 3HP fraction. ${ }^{122}$ Andreeßen and Steinbüchel explained this decreased crystallinity and increased biodegradability by the missing methyl group in the monomer's backbone. ${ }^{123} \mathrm{Kang}$ and colleagues discovered poly(3HBco-3HP) biosynthesis when supplementing the methylotrophic soil bacterium Methylobacterium sp. KCTC 0048 on methanol as carbon source plus the precursor $3 \mathrm{HP}{ }^{124}$ The first report on poly(3HB-co$3 \mathrm{HP}$ ) by recombinant bacteria was provided by Valentin et al., who supplied rec. E. coli expressing PHA synthesis genes (from $C$. necator) and genes encoding for propionyl-CoA synthetase (from Salmonella enterica) with 3HP; in this study, outstandingly high molar $3 \mathrm{HP}$ fractions in poly(3HB-co$3 \mathrm{HP}$ ) of up to $0.9 \mathrm{~mol} \mathrm{~mol}^{-1}$ were achieved when co-supplying mannitol and $3 \mathrm{HP}{ }^{125}$ In addition to $E$. coli, also $C$. necator was genetically engineered to make it produce poly(3HB-co-3HP) from structurally unrelated carbon sources. Fukui and colleagues introduced malonyl-CoA reductase and propionyl-CoA synthase from the $\mathrm{CO}_{2}$-fixation pathway of the green non-sulfur bacterium Chloroflexus aurantiacus. Via malonyl-CoA, 3HP-CoA was generated from acetyl-CoA in the recombinant strain. Poly (3HB-co-3HP) with 3HP fractions from 0.2 to $2.1 \mathrm{~mol} \%$ were obtained using fructose, gluconate or alkanoates (octanoate, dodecanoate). ${ }^{26}$ A later study demonstrated the first biosynthesis of poly(3HP) homopolyester, a material of typical thermoplastic properties; anaerobic Clostridium butyricum genes encoding for glycerol dehydratase, Salmonella enterica genes encoding for propionaldehyde dehydrogenase, and C. necator PHA synthase genes were expressed in rec. E. coli. Up to 12 wt. $\%$ poly $(3 \mathrm{HP})$ were obtained in a two-step fedbatch fermentation process using glycerol as sole carbon source. Importantly, the second stage of the process (PHA accumulation) had to be carried out under anaerobic conditions due to the nature of the glycerol dehydratase, which resulted in rather low productivity. ${ }^{127}$ Considerably higher poly(3HP) yields were obtained by Wang et al. in optimized, entirely aerobic, 3-Liter bioreactor fed-batch fermentation setups; these authors used recombinant E. coli expressing the aerobic Klebsiella pneumonia genes encoding for glycerol dehydratase, propionaldehyde dehydrogenase from Salmonella typhimuri$u m$, and $C$. necator PHA synthase for glycerol-based cultivations. Up to $22 \mathrm{~g} \mathrm{~L}^{-1} \mathrm{CDM}$ and $10.1 \mathrm{~g} \mathrm{~L}^{-1}$ P3HP, corresponding to 0.46 g PHA per g CDM, were achieved by this experiment. ${ }^{128} \mathrm{~A}$ new poly(3HP) biosynthesis pathway to biosynthesize poly(3HP) homopolyester by rec. E. coli was developed by Wang et al. Malonyl-CoA reductase from Chloroflexus aurantiacus, E. coli propionyl-CoA synthetase and acetyl-CoA carboxylase, and C. necator PHA synthase were expressed by this recombinant strain. When using glucose as sole, unrelated carbon source, $\mathrm{CDM}$ and poly $(3 \mathrm{HP})$ mass fraction in CDM amounted to $1.32 \mathrm{~g} \mathrm{~L}^{-1}$ and 0.01 $\mathrm{g} \mathrm{g}^{-1}$, respectively. ${ }^{129}$ 
PHA heteropolyesters containing branched building blocks

Watanabe and colleagues described the production of PHA containing $\alpha$-methylated, hence, branched building blocks in genetically engineered $E$. coli cells harboring the PHA synthase of $A$. caviae. When cultivated on tiglic acid [(2E)-2-methylbut-2-enoic acid)], this strain accumulated a new copolyester of 3HB and 3-hydroxy-2-methylbutyrate $(3 \mathrm{H} 2 \mathrm{MB})$ with reduced $T_{m}, T_{o}$, melting enthalpy $\left(\delta H_{m}\right)$, and $T_{c}$ if compared to PHB homopolyester. Especially the decreased $T$ indicated that poly(3HB$c o-3 \mathrm{H} 2 \mathrm{MB}$ ) showed higher affinity towards crystallization than previously described scl-PHA copolyesters. ${ }^{130}$ Further pathway engineering of this strain carried out by Furutate and colleagues, by weakening its $\beta$-oxidation metabolism and inserting a propionyl-CoA transferase gene, enabled the production of another new class of PHA copolyesters: cultivation in tiglic acid and hexanoic acid resulted in accumulation of poly (3H2MB-co-3HHx); these copolyesters, consisting of both branched scl-PHA and linear $m c l$-PHA building blocks, revealed low $T_{g}$ values and totally lacking $T_{m}$, hence, these products show no crystallinity in contrast to poly(3HBco-3H2MB). ${ }^{131}$ In addition, the production of PHA harboring 3HB, 3HV, 3H2MV and 3-hydroxy-2methylbutyrate $(3 \mathrm{H} 2 \mathrm{MB})$ was reported by Dai and colleagues, who cultivated a mixed culture rich in glycogen-accumulating organisms with high phylogenetic similarity with Defluviicoccus vanus under anaerobic conditions in not nutrient-limited media; propionic acid and acetate were used as carbon sources, with higher propionic acid fractions favoring incorporation of the branched monomers. Depending on the fractions of the branched building blocks, the generated poly(3HB-co-3HV-co$3 \mathrm{H} 2 \mathrm{MB}-\mathrm{co}-3 \mathrm{H} 2 \mathrm{MV}$ ) copolyesters had a molecular mass of $390-560 \mathrm{~kg} \mathrm{~mol}^{-1}, T_{m}$ of $70-160{ }^{\circ} \mathrm{C}$, and $T_{g}$ between -8 and $0{ }^{\circ} \mathrm{C}$. Generally, the authors observed that incorporation of the branched monomers generated defects in the poly $(3 \mathrm{HB}-\mathrm{co}-3 \mathrm{HV})$ lattice, thus resulting in decreased crystallinity of poly(3HB-co-3HV-co-3H2MB-co-3H2MV) copolyesters. ${ }^{132}$

Copolyesters harboring 3-hydroxy-4-methylvalerate $(3 \mathrm{H} 4 \mathrm{MV})$ as another branched building block are still scarcely described in literature; however, these materials are considered auspicious for applications due to the fact that they are not prone to increasing crystallization with time ("aging") as is the case with PHB or poly(3HB-co-3HV). Up to now, production of $3 \mathrm{H} 4 \mathrm{MV}$-containing PHA was reported for wild type and genetically modified organisms. The first report on 3H4MV-containing PHA was provided by Tanadchangsaeng et al., who equipped a genetically engineered, PHA-negative $C$. necator strain with $A$. caviae PHA synthesis genes. Feeding the recombinant organism with fructose and 4-methylvaleric acid resulted in accumulation of poly (3HB-co-3H4MV) with $3 \mathrm{H} 4 \mathrm{MV}$ fractions in PHA up to $0.35 \mathrm{~mol} \mathrm{~mol}^{-1}$. These polyesters were well fractionable into randomly distributed copolyesters with $3 \mathrm{H} 4 \mathrm{MV}$ fractions up to $0.47 \mathrm{~mol}$ mol $^{-1}$; crystallinity, $T_{m}$ and $T_{\sigma}$ values strongly decreased with increasing $3 \mathrm{H} 3 \mathrm{H} V$ fractions, whereas susceptibility to enzymatic degradation increased in parallel. ${ }^{133}$ Similar attempts were reported by Chia and colleagues, who equipped the same PHA-negative C. necator with Chromobacterium sp. USM2 PHA synthase genes. Feeding this recombinant strain with palm kernel oil and 4-methylvaleric acid resulted in accumulation of $s c l$ - $m c l$-PHA copolyesters containing $3 \mathrm{HB}, 3 \mathrm{HV}, 3 \mathrm{HHx}$ and $3 \mathrm{H} 4 \mathrm{MV}$. Exemplarily investigating films of one of the products, poly(3HB-co-1 mol\% $3 \mathrm{HV}-\mathrm{co}-3 \mathrm{~mol} \% 3 \mathrm{H} 4 \mathrm{MV}-$ co-18 mol\% $3 \mathrm{HHx}$ ) revealed excellent rubber-like properties of this material in terms of higher thermal stability, higher elasticity, outstanding flexibility, and higher ductility if compared with poly $(3 \mathrm{HB})$ and even poly(3HB-co-3HHx). ${ }^{134}$ The wild type strain Chromobacterium sp. USM2, donator of the PHA synthase in the study of Chia et al.,${ }^{134}$ was also directly used by Ling et al. to investigate production of 3H4MV-containing PHA. These authors reached a molar $3 \mathrm{H} 4 \mathrm{MV}$ fraction of $22 \%$ when using 4-methylvaleric acid as sole carbon source. In addition, these authors found that decreased oxygen concentration in the cultivation medium was beneficial for poly(3HB-co-3H4MV) accumulation without drastically affecting cell growth at the same time. ${ }^{135}$ Later, Saika and associates remarked that small amounts $(0.5 \mathrm{~mol} \%)$ of $3 \mathrm{H} 4 \mathrm{MV}$ were produced by PHA-negative $C$. necator harboring Pseudomonas sp. 61-3 PHA synthase when cultured on fructose as sole carbon source. Co-feeding with excess amounts of the branched amino acid leucine as $3 \mathrm{H} 4 \mathrm{MV}$ structurally related precursor compound was found to increase the production of $3 \mathrm{H} 3 \mathrm{MV}$. Even higher fractions of this branched monomer were obtained after manipulating the leucine metabolism of the production strain by manipulating the leucine feedback inhibition; up to $3.1 \mathrm{~mol} \% 3 \mathrm{H} 3 \mathrm{MV}$ plus $1.2 \mathrm{~mol} \% 3 \mathrm{HV}$ were obtained by this new engineered strain. Again, the copolyester displayed enhanced thermal properties in comparison to PHB or poly (3HB-co-3HV) due to decreased crystallinity. ${ }^{136}$ Lau and colleagues investigated the wild type organism Burkholderia sp. USM and its transformant harboring Aeromonas caviae PHA synthase gene for $3 \mathrm{H} 4 \mathrm{MV}$ formation by cultivations on hexoses and 4-methylvaleric acid. Whereas the wild type produced only minor amounts (up to $1 \mathrm{~mol} \%$ ) 
of $3 \mathrm{H} 4 \mathrm{MV}$ in poly(3HB-co-3H4MV) copolyesters, up to $40 \mathrm{~mol} \%$ of $3 \mathrm{H} 4 \mathrm{MV}$ were reached in the transformed organism. Moreover, the authors demonstrated that $3 \mathrm{H} 4 \mathrm{MV}$-containing PHA is intracellularly mobilized by the strain by culturing copolyester-rich cells in carbon-free medium. ${ }^{137}$

\section{PHA heteropolyesters containing other unusual building blocks}

The first report on PHA harboring aromatic building blocks was provided in 1991 by Kim et al., who supplied $P$. oleovorans with mixtures of 5 -phenylvaleric acid and the alkanoic acids $n$-nonanoic acid or $n$-octanoic acid, respectively. When using 5-phenylvaleric acid parallel with $n$-nonanoic acid, odd-numbered 3-hydroxyalkanoates (C5-C11) and $3 \mathrm{H} 5 \mathrm{PV}$ building blocks were detected; in the case of using octanoic acid plus 5-phenylvaleric acid, even numbered 3-hydroxyalkanoates (C6-C10) were found parallel with $3 \mathrm{H} 5 \mathrm{PV}$. It was shown that the fraction of aromatic building blocks drastically increased with increasing concentrations of 5-phenylvaleric acid in the substrate feed. Surprisingly, the authors provided no comment on the fact of having detected building blocks longer than the provided substrates. ${ }^{138}$

Moreover, the occurrence of PHA building blocks with diverse functional groups other than unsaturated or aromatic groups was described years ago for halogenated PHA or PHA harboring epoxy-, ester-, or cyano groups. In each of these exotic cases, $P$. putida acted as production strain. PHA with 9-cyano-3-hydroxynonanoate and 7-cyano-3-hydroxyheptanoate as monomers were obtained by co-feeding $n$-octanoic or $n$-nonanoic acid and 11-cyanoundecanoic acid as carbon sources, ${ }^{139}$ whereas bromated PHA was produced using nonanoic or octanoic acid and 6-bromohexanoic acid, 8-bromo-octanoic acid or 11-bromoundecanoic acid as substrate for P. oleovorans. ${ }^{140}$ Chlorinated PHA was produced using the same organism by Doi and Abe, who applied mixtures of octane and 1-chlorooctane as carbon source in bioreactor cultivations. According to ${ }^{1} \mathrm{H}$ - and ${ }^{13} \mathrm{C}-\mathrm{NMR}$ analysis, a random copolyester containing $3 \mathrm{HHx}, 3 \mathrm{HO}, 6$-chloro-3-hydroxyhexanoate and 8-chloro-3-hydroxyoctanoate was produced. This material turned out to be completely amorphous; no melting endotherms were observed in the respective thermograms. $M_{n}$ of this new PHA amounted to only $87 \mathrm{kDa}^{141}$ The same research group reported also on production of $\omega$-fluorinated PHA building blocks when cultivating $P$. putida on mixtures of nonane and 1-fluorononane. ${ }^{142}$ Later, these scientists improved this process by substituting nonane with gluconic acid; thus, it was possible to produce new PHA copolyesters in a controlled manner with a molar fraction of fluorinated build- ing blocks between 0 and $40 \%$. The materials revealed melting temperature between 50 and $60{ }^{\circ} \mathrm{C}$, and showed deceasing melting enthalpy with increasing fluorinated fraction. ${ }^{143}$ Using a mixture of 10-epoxyundecanoic acid and sodium octanoate as substrate enabled the formation of PHA with pendent epoxy groups; relative high molecular mass ( $M_{n}$ about $140 \mathrm{kDa}$ ), and very low $T_{g}$ values ( -30 to $-60^{\circ} \mathrm{C}$ ) were determined for these novel polyesters. These materials can further undergo chemical modification, e.g., towards carboxy groups, which provides PHA of outstanding hydrophilicity, making it even water-soluble. ${ }^{144}$ It has to be emphasized that those exotic PHAs were produced at low productivity, and only tiny amounts of these polymers were de facto generated; based on the high costs for the required functionalized co-substrates, no significant activities were reported over the last two decades on such unusual biopolymers. By chemical means, PHA bearing epoxide groups were produced by post-synthetic conversion of terminal alkene groups of unsaturated $\mathrm{mcl}$-PHA in order to facilitate crosslinking of these polymers, ${ }^{132}$ or the terminal groups were oxidized to diol ${ }^{145}$ or carboxyl ${ }^{146}$ groups to increase the hydrophilicity of the products. Scholz and colleagues utilized methyl, ethyl, and tert-butyl esters of octanoic, nonanoic and decanoic acid as feedstocks for $P$. putida. All applied substrates resulted in accumulation of PHA copolyesters with up to eight different monomers. Especially methyl esters gave copolyesters with a high degree of monomers with pendent methyl ester groups, while ethyl esters resulted in generation of copolyesters with unsubstituted monomers, monomers with pendant methyl- and ethyl ester groups. In contrast, the tert-butyl ester group was found in the generated PHA samples. Remarkably, the authors elucidated that the fraction of ester groups found in the polymers strongly depended on the oxygen supply; while restricted oxygen availability resulted in conversion of the substrate's carboxylate group rather than in formation of a new carboxylate group, hence to formation of 3-hydroxyalkanoates, excess supply of oxygen resulted in an attack of the substrate's alkane end, thus preserving the carboxylate group, therefore found in the polyesters. ${ }^{147}$

\section{PHA quarterpolyesters}

As a rather new class of PHA, so-called "PHA quarterpolymers" were recently reported by Zhila and Shishatskaya. ${ }^{148}$ This group of heteropolyesters contains four different types of monomers, namely chiral scl-PHA building blocks with different side chains (3HB and $3 \mathrm{HV}$ ), an achiral scl-monomer (4HB), and a $m c l$-PHA building block (3HHx). Using C. eutrophus B10646 as production strain in twostage batch cultivation setups, the physicochemical, 
mechanical, and biological properties of poly $(3 \mathrm{HB}-$ co-3HV) copolyesters $(10.4-75.0 \mathrm{~mol} \% 4 \mathrm{HB}$; in the publication: "bi-polymers"), poly( $3 \mathrm{HB}-\mathrm{co}-3 \mathrm{HV}-$ co-4HB) terpolyesters $(28.7-55.6 \mathrm{~mol} \% 4 \mathrm{HB})$, and poly (3HB-co-3HV-co-4HB-co-3HHx) quarterpolyesters $(9.3-13.3 \mathrm{~mol} \%$ 4HB) were studied. Glucose acted as main carbon source for all produced polyesters, valeric acid, GBL, and hexanoic acid were used as precursors for $3 \mathrm{HV}, 4 \mathrm{HB}$, and $3 \mathrm{HHx}$ production, respectively. By comparison with PHB homopolyester prepared in the same experimental series, it was shown that increasing fractions of $4 \mathrm{HB}$ improved the processability of the polyesters by lowering crystallization temperature, melting point, and crystallinity, and by increasing the flexibility of the material, expressed as elongation at break. These beneficial effects were enhanced by the presence of $3 \mathrm{HV}$ in ter- and quarterpolymers, and by $3 \mathrm{HHx}$ in quarter-polymers. Remarkably, all heteropolyesters had significantly lower molecular mass $\left(M_{w}\right.$ between 540 and $\left.830 \mathrm{kDa}\right)$ than the homopolyester $\left(M_{w}\right.$ almost $\left.1 \mathrm{MDa}\right)$, but almost identical thermal stability. Films produced from the different PHA samples showed similar microstructure and porosity; none of them revealed any toxic effect on fibroblasts, thus underlining the biocompatibility of these materials. As shown previously, co- and terpolyesters containing, beside $3 \mathrm{HB}$, one or two of the monomers $3 \mathrm{HV}, 4 \mathrm{HB}$ and $3 \mathrm{HHx}$, can also be produced by this strain under mixotrophic cultivation conditions by replacing glucose with $\mathrm{CO}_{2}$ in a recycled-gas closed-circuit cultivation system. ${ }^{248}$

\section{Heteropolyesters containing 2-hydroxy building blocks}

Apart from 3- and 4-hydroxyalkanoic acids (3HA and 4HA, respectively), 2-hydroxyalkanoic acids (2HA) with various side chains were recently reported as PHA building blocks when using genetically engineered $E$. coli strains harboring the lactate polymerizing enzyme (LPE). After sophisticated pathway design, cells expressing LPE, (R)-2-hydroxy-4-methylvalerate (2H4MV) dehydrogenase (LdhA) and 2H4MV-CoA transferase (HadA) from Clostridium difficile plus $\mathrm{mcl}$ - and $\mathrm{scl}$ PHA synthesis genes from Pseudomonas sp. 61-3 and $C$. necator $\mathrm{H} 16$, respectively, were found to accumulate poly(3HB-co-2HA) from both related or unrelated carbon sources. 2HA monomers found in PHA from unrelated substrates (glucose, xylose, glycerol) were primarily $2 \mathrm{H} 4 \mathrm{MV}$ and the aromatic building block 2-hydroxy-3-phenylpropionate $(2 \mathrm{H} 3 \mathrm{PhP})$, which were assumed to be generated from intracellular pools of leucine and phenylalanine, respectively. In addition, 2-hydroxypropionate (2HP), 2-hydroxy-3-methylbutyrate (2H3MB) and 2-hydroxy-3-methylvalerate (2H3MV) were identi- fied as building blocks of these remarkable PHA samples. Structurally related amino acids as precursors for individual 2HA were supplemented; here, it was shown that supplementation with leucine increased the level of $2 \mathrm{H} 4 \mathrm{MV}$, supplementation of valine increased the level of $2 \mathrm{H} 3 \mathrm{MB}$, and phenylalanine boosted the fraction of the aromatic monomer $2 \mathrm{H} 3 \mathrm{PhP}$. Further experiments were carried out with saccharified cedar wood as an abundant lignocellulose feedstock; also in this case, poly(3HB-co-2HA) with more than 30 mol- $\%$ 2HA was successfully accumulated. ${ }^{149}$

\section{mcl-PHA homopolyesters}

In vivo synthesis of $m c l$-PHA homopolyesters to date is described exclusively with the use of genetically engineered microorganisms. ${ }^{150}$ In most cases, $m c l$-PHA are synthesized starting with $\beta$-oxidation of fatty acids (vide supra), which produces a cocktail of 3-hydroxyalkyl-CoAs, encompassing 3-hydroxyhexanoyl-CoA and other monomers with longer side chains, with the maximum length of monomers depending on the chain length of the metabolized fatty acid. ${ }^{88} \beta$-oxidation-weakened mutants of $P$. putida were generated by Liu and colleagues, who knocked out the genes encoding for the key enzymes catalyzing fatty acid degradation and for the 3-hydroxyacyl-CoA-acyl carrier protein. When supplied with decanoic acid as a carbon source, the obtained $\beta$-oxidation-weakened mutant strain successfully accumulated poly(3-hydroxydecanoate) homopolyester, while poly(3HDco-84 \%-3HDD) was generated when feeding with dodecanoic acid; this is in contrast to the wildtype strain, which produces copolyesters composed of three $(3 \mathrm{HHx}, 3 \mathrm{HO}, 3 \mathrm{HD})$ or four $(3 \mathrm{HHx}, 3 \mathrm{HO}$, 3HD, 3HDD) different monomers, respectively. ${ }^{151}$ Applying this $\beta$-oxidation weakening approach to Pseudomonas entomophila L48, similar results were reported. Using the new strain $P$. entomophila LAC23, an almost pure (3HDD molar fraction $>99$ \%) poly(3HDD) homopolyester was obtained from dodecanoic acid, with polymer fractions in biomass exceeding $0.9 \mathrm{~g} \mathrm{~g}^{-1} .{ }^{152}$ Later, Wang et al. used this organism for cultivations on defined C7-C14 fatty acids (heptanoic acid, octanoic acid, nonanoic acid, decanoic acid, undecanoic acid, dodecanoic acid, tridecanoic acid, and tetradecanoic acid) as sole carbon sources in order to obtain the homopolyesters of corresponding 3HAs. Although productivities were rather modest due the high toxicity of the substrates, the authors succeeded in producing the desired homopolyesters in almost pure form; only traces of monomers shortened by two carbon atoms due to $\beta$-oxidation were detected, which allowed them to study the properties of different $m c l$-PHA homopolyesters for the very first time. As a remark- 
able finding, it was shown that $T_{g}$ and thermal stability of $m c l$-PHA homopolyesters of odd-numbered monomers $(\mathrm{C} 7, \mathrm{C} 9, \mathrm{C} 11, \mathrm{C} 13)$ by trend increase with increasing number of carbon atoms in the monomer, while the opposite trend was observed for homopolyesters with even number of carbon in monomers. Independent of the monomers being odd or even, $T_{m}$ increased with increasing side chains of monomers. The authors emphasized that, after hydrolysis of these homopolyesters, pure chiral bioactive monomers can be obtained, which are of high pharmaceutical value. ${ }^{153}$

\section{Microstructure of PHA: Random distribution vs. Blocky structure}

In addition to the monomeric composition, the microstructure of PHA is another decisive parameter for the material properties of the biopolymer. Generally, blocky structured PHA heteropolyesters (b-PHA), PHA heteropolyesters with monomers randomly distributed, and polymer blends can occur in living cells (see Fig. 5).

Chains of $b$-PHA consist of at least two distinctive, covalently linked polymer sections (blocks). Properties of each block are encompassed by this structure, which opens the door to new polymer properties, not accessible by simple blending of polymers with the structure of the individual bocks. In a nutshell, production of $b$-PHA offers the possi- bility of biopolyesters with tunable, pre-defined microstructure. Moreover, $b$-PHA are reported to be less prone to polymer aging. ${ }^{154}$ Examples of $b$-PHA copolyester structures are diblocks (e.g., $[3 \mathrm{HB}]_{\mathrm{x}}-$ $\left.[3 \mathrm{HV}]_{\mathrm{y}}\right)$, triblocks (e.g., $[3 \mathrm{HB}]_{\mathrm{x}}-[3 \mathrm{HV}]_{\mathrm{y}}-[3 \mathrm{HB}]_{\mathrm{z}}$ or $\left.[3 \mathrm{HB}]_{\mathrm{x}}-[3 \mathrm{HV}]_{\mathrm{V}}-[4 \mathrm{HB}]_{\mathrm{z}}\right)$, or repeating multiblocks (e.g., $\left.[3 \mathrm{HB}-3 \mathrm{HV}]_{n}\right) .{ }^{155}$ Apart from microbial biosynthesis, traditional chemical synthesis techniques can be applied to generate artificial $b$-PHA, as shown when linking blocks of atactic PHB (a-PHB), a material obtained by ring opening polymerization of $\beta$-butyrolactone, to blocks of poly(6-hydroxyhexanoate). ${ }^{156}$ In addition, polyesterurethanes (PEU) consisting of "hard" (PHB) and "soft" (PHO) PHA segments, covalently linked by diisocyanate, or PHB blocks linked to poly(ethylene glycol) blocks were generated. Via racemic polymerization, amphiphilic triblock copolymers, such as poly(a-PHB$b$-ethylene glycol- $b$-a-PHB) are accessible, which overcome the insufficient hydrophilicity of a-PHB ${ }^{157}$ More recently, novel diblock copolymers of different types of PHA (PHB, PHBV, PHO) and a-PHB were generated, which hold high promise as blend compatibilizers for cardiovascular engineering. ${ }^{158}$

In addition to chemical synthesis of PHA-based block polymers, $b$-PHA is also directly accessible by adapting the biotechnological production strategy, although PHA heteropolyesters are typically synthe-

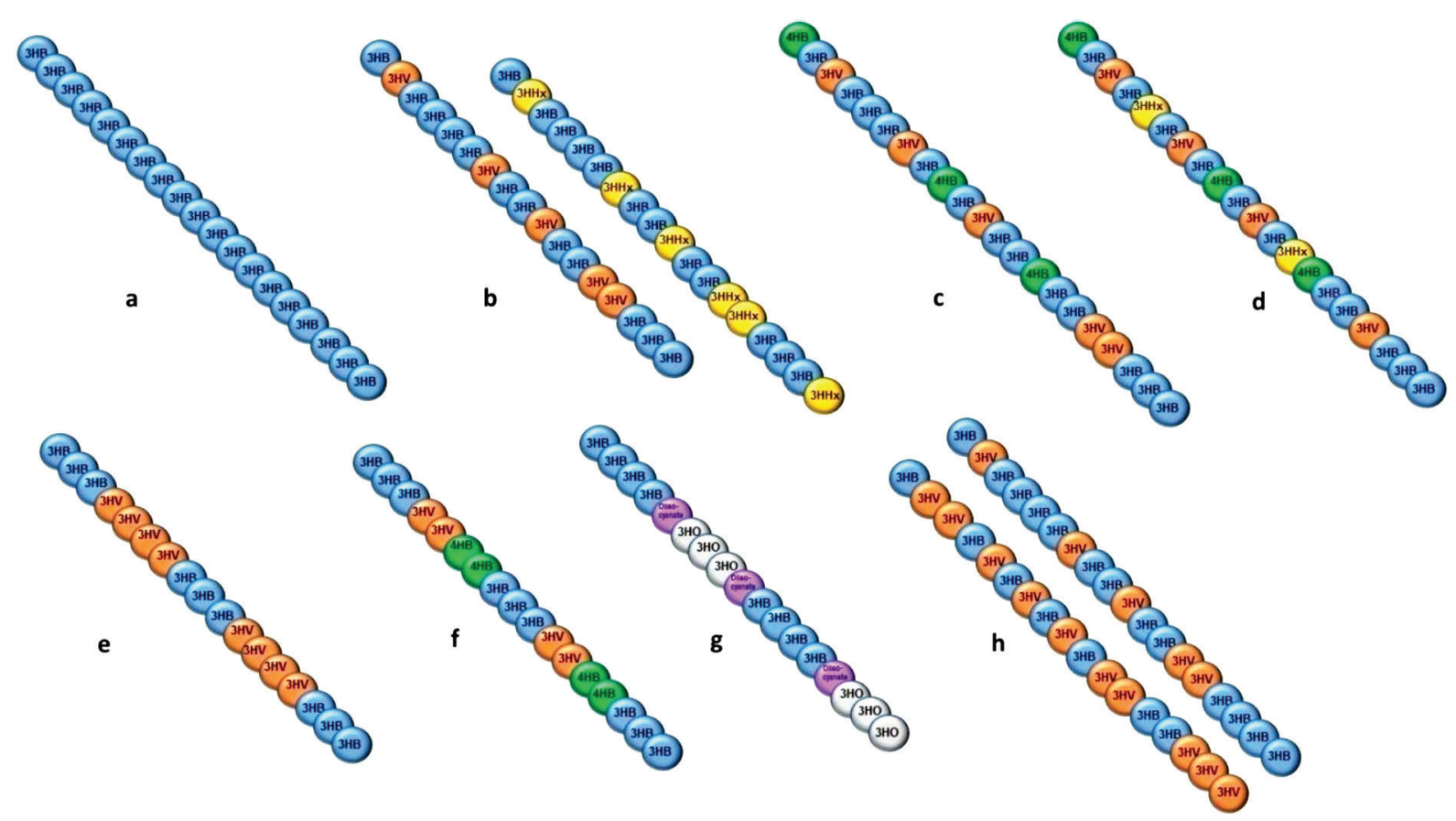

Fig. 5 - Schematic of PHAs of different microstructure. Blue spheres: 3HB; orange spheres: $3 H V$; green spheres: 4HB; yellow spheres: $3 H H x$; grey spheres: $3 H O$; pink spheres: linker diisocyanate. a: PHB homopolyester; $b$ : copolyesters with random distribution (left: poly(3HB-co-3HV), right "Nodax-type" poly(3HB-co-3HHx)); c: poly(3HB-co-3HV-co-4HB) terpolyester with random distribution; d: poly( $3 H B-c o-3 H V-c o-4 H B-c o-3 H H x)$ quarterpolyester with random distribution; e: diblock b-PHA poly (PHB-b-PHV); f: triblock b-PHA poly(PHB-b-PHV-b-P4HB); g: polyesterurethane (PEU) with "hard" PHB and "soft" PHO blocks linked by diisocyanate; $h$ : PHA blend of $3 H V$-rich and $3 H V$-poor poly $(3 H B-c o-3 H V)$ randomly distributed copolyesters. 
sized randomly with arbitrary interspaces of the individual monomers along the PHA chain, which makes control or predefinition of polyester properties difficult. In this context, Pederson and co-workers described the impact of adjusting the supply of C. necator $\mathrm{H} 16$ with fructose and the $3 \mathrm{HV}$ precursor valeric acid. It was shown that parallel availability of fructose and valeric acid, realized by permanent supply of fructose and periodic valerate pulse feeding after its exhaust, generated poly(3HB-co$3 \mathrm{HV})$ copolyesters of random distribution, whereas during periods of fructose-only availability, PHB homopolyester blocks were generated. Now, the authors developed a feedback-controlled bioreactor cultivation system, which triggered the valerate supply as response to DO in fermentation broth and to $\mathrm{CO}_{2}$ concentration in the exhaust air stream from the bioreactor, and succeeded in generating poly(PHB- $b$-PHBHV) diblock copolyesters of defined length of the individual blocks. ${ }^{155}$

A recent study provided by Ferre-Guell and Winterburn investigated cultivation strategies for the haloarcheon $H f x$. mediterranei to produce randomly distributed and blocky structured poly(3HBco-3HV) copolyesters. ${ }^{159}$ Cultures of these organisms were supplied with different VFAs of even (acetic, butyric, hexanoic, octatonic, and decanoic acid) and odd (propionic, valeric, heptanoic, nonanoic, undecanoic acid) number of carbon atoms. Growth and PHA production were observed only for those VFAs with less than seven carbon atoms. While acetic acid generated poly $(3 \mathrm{HB}-\mathrm{co}-3 \mathrm{HV})$ with about $10 \mathrm{~mol} \% 3 \mathrm{HV}$ (similar to setups on glucose), the $3 \mathrm{HV}$ fraction in polyesters produced from butyric acid was negligible. Using valeric acid as sole carbon source, copolyesters with outstandingly high (more than $90 \mathrm{~mol} \%$ ) $3 \mathrm{HV}$ content were obtained, whereas $3 \mathrm{HV}$ fraction in polyesters from propionic acid was lower due to a partial oxidative decarboxylation of propionyl-CoA towards the $3 \mathrm{HB}$ precursor acetyl-CoA, which is in accordance with the findings discussed previously. Different feeding strategies were carried out using butyric and valeric acid or defined mixtures thereof; it was found that sequential feeding of one VFA after the other generated blocky structured copolyesters, whereas co-feeding resulted in random distribution of the building blocks, as evidenced by ${ }^{1} \mathrm{H}$ and ${ }^{13} \mathrm{C}$ NMR measurements. Regarding the thermal properties of the copolyester samples, it was shown that increasing $3 \mathrm{HV}$ fraction in randomly distributed polymers resulted in a decrease in $T$, thus in a higher mobility of PHA chains in the amorphous phase. General$1 \mathrm{y}$, increasing $3 \mathrm{HV}$ fractions in random poly $(3 \mathrm{HB}-$ co-3HV) samples favored the amorphous character of the polymers, as evidenced by lacking $T_{c}$ peaks in DSC thermograms. Moreover, $3 \mathrm{HV}$-rich copolyes- ters exhibited lower $T_{m}$, improved elasticity, and enhanced ductility. ${ }^{159}$

As shown by Ashby et al., sequential feeding of substrates to generate $b$-PHA can be overcome by using substrates that are not converted in parallel by the production strain, which results in a kind of "diauxic PHA biosynthesis". The authors used $B$. sacchari DSM 17165, a currently emerging PHA production strain (vide supra), on substrate mixtures of xylose and levulinic acid. ${ }^{112}$ As shown previously, xylose, as follow-up material of inexpensive lignocelluloses, was converted by this strain for PHB homopolyester production, ${ }^{160}$ whereas levulinic acid, also accessible as platform chemical starting from renewable resources, performed successfully as $3 \mathrm{HV}$ precursor for other PHA-producing microbes. ${ }^{11,112}$ The study reported by Ashy et al. demonstrated that, when supplying both substrates in parallel, levulinic acid was converted preferentially to xylose by B. sacchari. By adapting the initial concentration of levulinic acid in the substrate mix and the fermentation length, the size of poly $(3 \mathrm{HV})$ blocks in poly $(3 \mathrm{HB}-b-3 \mathrm{HV})$ diblock copolyesters can be varied. Remarkably, these polyesters revealed outstandingly high molecular masses exceeding 1 MDa. ${ }^{112}$

As shown over the last couple of years, PHA can be produced at high productivity in continuously operated multistage bioreactor cascades; ${ }^{161}$ these processes are well-investigated by diverse approaches of mathematical modelling. ${ }^{162-165}$ Such bioreactor cascade systems are not only suggested for high-throughput PHA biosynthesis, but, although up to now investigated experimentally only for PHB production, also offer the possibility of feeding individual cascade stages with different carbon sources, thus generating designed PHA blocks. This approach requests complete consumption of a carbon source in a given stage of the cascade, and subsequent feeding of another carbon source in the next stage, etc. ${ }^{161}$ This process engineering tool might be a decisive leap in direction of producing tailor-made PHA of pre-defined composition and properties.

When mcl-PHA is generated by Pseudomonades using $\beta$-oxidation of fatty acids, they typically display random distribution of the building blocks; this was recently demonstrated by Impallomeni and colleagues, who produced randomly distributed mcl-PHA copolyesters with building blocks up to $3 \mathrm{HHpD} .{ }^{100}$ Other researchers purposely aimed at production of randomly distributed copolyesters. In this context, mixotrophic cultivations in a 7-liter bubbled and stirred tank bioreactor was investigated by Ghysels and colleagues; $\mathrm{CO}_{2}$ and valeric acid were metabolized by the well-described "Knallgasbacterium" C. necator DSM 545 towards poly(3HB- 
co-3HV) copolyesters of tailored composition and monomeric distribution. $\mathrm{CO}_{2}$ was continuously sparged, whereas valeric acid was added as substrate pulses and by $\mathrm{pH}$-stat feeding; thus, copolyesters containing more than $60 \mathrm{~mol} \% 3 \mathrm{HV}$ were obtained. As confirmed by ${ }^{1} \mathrm{H}$ and ${ }^{13} \mathrm{C}$ NMR, the desired random monomer distribution can be obtained by keeping the valeric acid concentration below $1 \mathrm{~g} \mathrm{~L}^{-1}$, as shown by the values for dyad sequence distribution and the degree of randomness both being close to 1 . Based on validation by independent datasets, the authors developed a reproducible and highly predictive model for mixotrophic production of poly (3HB-co-3HV) of pre-defined composition. ${ }^{166}$ Also regarding poly $(3 \mathrm{HB}-c o-3 \mathrm{HP})$ copolyesters reported previously herein, NMR measurements revealed that the parallel supply of $C$. necator and A. latus with sucrose and $3 \mathrm{HP}$ resulted in formation of copolyesters of random distribution. ${ }^{121}$

A PHA copolyester reported by Cespedes et al., produced using a genetically engineered Pseudomonas sp. from glucose, was found to consist of a single copolyester of two polymer blocks: the first block revealed the typical composition of a $\mathrm{mcl}$ PHA from other described Pseudomonas sp., whereas the second block exhibited the properties of Nodax-type scl-mcl-copolyesters, containing 3HB and $3 \mathrm{HHx}$ with a high molar $3 \mathrm{HHx}$ fraction. These unexpected findings shed new light on the substrate specificity of Aeromonas sp. PHA synthase. ${ }^{167}$ Blocky structured PHA consisting of "soft" and "hard" segments were generated using genetically engineered $E$. coli. Sequential feeding with 1,4-butanediol (precursor for the "soft" segment poly $(4 \mathrm{HB})$ ) and 1,3-propanediol (precursor of the "hard" segment poly(3HP)) resulted in biosynthesis of poly(poly(4HB)- $b$-poly(3HP)). The authors underlined the convenient properties of these blocky structured polyesters in comparison to randomly distributed analogues regarding lower melting points, and drastically better and higher Young's modulus, yield strengths, and tension strengths. Typical for $b$-PHA, the new materials displayed two $T_{g}$ and two $T_{m}$ peaks. ${ }^{168}$

The $\beta$-oxidation weakened $P$. entomophila mutant strain described by Wang et al. for mcl-PHA homopolyester production (vide supra), was cultivated on mixtures of octanoate and dodecanoic acid or tetradecanoic acid, respectively. Depending on the composition of the substrate mix and the cultivation time, fractions of poly(3HO-co-3HDD) and poly(3HO-co-3HTD) copolyesters of random distribution and adaptable 3HDD or 3HTD, respectively, content were obtained; only traces of other monomers (3HHx or $3 \mathrm{HD}$ ) were detected due to the $\beta$-oxidation deficiency of the strain. In the same study, the authors demonstrated sequential feeding as a viable tool to generate blocky structured poly(3HO- $b-3 \mathrm{HDD})$ copolyesters by this organism by adding the second substrate (dodecanoic acid) only after depletion of the first (octanoate). ${ }^{153} \mathrm{~A}$ similar approach was used by Li et al., who cultured recombinant $P$. putida harboring Aeromonas caviae PHA synthesis genes (P. putida KTQQ20) on butyrate and heptanoate in a 5-L bioreactor. Again, sequential feeding (addition of heptanoate only after depletion of butyrate) resulted in the production of a new class of $b$-PHA, namely a copolyester containing alternating blocks of PHB homopolyester and random poly( $3 \mathrm{HV}-\mathrm{co}-3 \mathrm{HHp})$; $3 \mathrm{HV}$ monomers were generated by the loss of one acetyl-CoA unit via $\beta$-oxidation. Feeding a mixture of butyrate and heptanoate resulted in formation of randomly distributed poly (3HB-co-3HV-co-3HHp) copolyesters. The authors underlined the expedient material quality of the $b$-PHA by comparing its tensile strength and Young's modulus with data obtained from the randomly distributed copolyester and with a PHA blend of PHB and poly $3 \mathrm{HV}-\mathrm{co}$ $3 \mathrm{HHp}$ ) of similar overall monomeric composition. ${ }^{169}$ Later, two different $\beta$-oxidation weakened $P$. putida mutants were used for biosynthesis of $m c l$-PHA with tunable composition and microstructure. When cultivated on butyrate/hexanoate mixtures, poly(3HB-co-3HHx) copolyesters with random distribution were accumulated by $P$. putida KTOYO6 $\Delta \mathrm{C}$, whereas using hexanoate/decanoate mixtures resulted in biosynthesis of randomly distributed poly $(3 \mathrm{HHx}-\mathrm{co}-3 \mathrm{HD})$ copolyesters by $P$. putida KTQQ20. The monomeric compositions were easily tunable by varying the ration of fatty acids in the feed. In contrast, sequential feeding of P. putida KTQQ20 with hexanoate and dodecanoate resulted in production of a novel diblock copolymer $\operatorname{poly}(\mathrm{P}(3 \mathrm{HHx})-b$-poly(3HD-co-3HDD $)) .{ }^{170}$

The latest discovery in the field of $b$-PHA is the production of poly $(2 \mathrm{HB}-b-3 \mathrm{HB})$ by a recombinant $E$. coli harbouring an engineered enzyme designated as $\mathrm{PhaC}_{\mathrm{AR}}$, which was a chimeric synthase of $A$. caviae and $C$. necator PHA synthases. Cultivation was performed by parallel availability of the two substrates sodium $(R, S)$-3HB and sodium $(R, S)$ $2 \mathrm{HB}$ at varied concentrations. ${ }^{1} \mathrm{H}$ NMR ruled out the possibility of the polymer being a randomly distributed copolyester, while subsequent solvent (THF) fractionation studies confirmed that, de facto, a $b$-PHA was produced with the blocks of PHB and poly $(2 \mathrm{HB})$ being covalently linked. The authors assume rapid intracellular changes of 2HB-CoA and $3 \mathrm{HB}-\mathrm{CoA}$ pools and fluctuating individual availability of these thioesters being responsible for formation of $b$-PHA instead of a random copolyester by this chimer synthase. ${ }^{171}$ 
A typical intracellular blend of PHA was obtained for the products produced by Kim and Lenz with $P$. putida on alkanoic acids and 5-phenylvaleric acid (vide supra). Based on fractionation studies and the comparison of $T$ and $T_{m}$ of the products with values for poly $(3 \mathrm{HN})$ and poly $(3 \mathrm{HO})$, respectively, it became clear that two different polymers were present in the cells in parallel, namely, a fraction rich in $3 \mathrm{H} 5 \mathrm{PV}$ and a fraction poor in this aromatic compound. ${ }^{138}$ Much later, the production of PHA blends by Hfx. mediterranei was demonstrated by Don et al., who fractionated poly (3HB-co-3HV) produced by this organism from glucose and yeast extract with chloroform/acetone. The major fraction (about $93 \%$ ) contained $10.7 \% 3 \mathrm{HV}$ and was of high molecular mass of $570 \mathrm{kDa}$, whereas the smaller fraction had a higher $3 \mathrm{HV}$ fraction $(12.3 \%)$ and a considerably low molecular mass of $78 \mathrm{kDa} \cdot{ }^{172}$ These results were later confirmed by Koller et al., who extracted lyophilized $H f x$. mediterranei biomass containing a poly (3HB-co-3HV-co-4HB) copolyester with acetone in a Soxleth apparatus; also in this case, a low molecular mass fraction (209 $\mathrm{kDa}$, not even $1 \%$ of the entire PHA on a mass basis) was extracted, while the major part of PHA (molecular mass of more than $1 \mathrm{MDa}$ ) remained in the biomass, and was acetone-extracted only under pressure and temperature above acetone boiling point. ${ }^{38}$

\section{Conclusions}

This review shows that PHA material properties strongly depend on the monomeric composition of these biopolyesters, and on their microstructure. Different microbiological, synthetic biological, process engineering, and biosynthetic approaches are available to manufacture tailor-made PHA according to individual application needs; thus, the properties of a considerable part of currently produced and applied plastics can be mimicked by biological alternatives. A huge variety of differently composed PHA with pre-defined material performance will be available in a not too distant future, enabling the already long awaited substitution of various petrol-based plastics contemporarily governing the polymer market.

\section{References}

1. Geyer, R., Jambeck, J. R., Law, K. L., Production, use, and fate of all plastics ever made, Sci. Adv. 3 (2017) e1700782. doi: https://doi.org/10.1126/sciadv.1700782

2. Singh, N., Hui, D., Singh, R., Ahuja, I. P. S., Feo, L., Fraternali, $F$., Recycling of plastic solid waste: A state of art review and future applications, Composites Part B: Eng. 115 (2017) 409.

doi: https://doi.org/10.1016/j.compositesb.2016.09.013
3. Koller, M., Braunegg, G., Advanced approaches to produce polyhydroxyalkanoate (PHA) biopolyesters in a sustainable and economic fashion, The EuroBiotech Journal 2 (2018) 89. doi: https://doi.org/10.2478/ebtj-2018-0013

4. Lebreton, L. C., Van der Zwet, J., Damsteeg, J. W., Slat, B., Andrady, A., Reisser, J., River plastic emissions to the world's oceans, Nat. Commun. 8 (2017) 15611. doi: https://doi.org/10.1038/ncomms15611

5. Braunegg, G., Bona, R., Koller, M., Sustainable polymer production, Polymer Plast. Technol. Eng. 43 (2004) 1779. doi: https://doi.org/10.1081/PPT-200040130

6. Rigamonti, L., Grosso, M., Møller, J., Sanchez, V. M., Magnani, S., Christensen, T. H., Environmental evaluation of plastic waste management scenarios, Resour. Conserv. Recy. 85 (2014) 42.

doi: https://doi.org/10.1016/j.resconrec.2013.12.012

7. Kourmentza, C., Plácido, J., Venetsaneas, N., Burniol-Figols, A., Varrone, C., Gavala, H. N., Reis, M. A., Recent advances and challenges towards sustainable polyhydroxyalkanoate (PHA) production, Bioengineering 4 (2017) 55. doi: https://doi.org/10.3390/bioengineering4020055

8. Koller, M., Maršálek, L., Miranda de Sousa Dias, M., Braunegg, G., Producing microbial polyhydroxyalkanoate (PHA) biopolyesters in a sustainable manner, New Biotechnol. 37 (2017) 24. doi: https://doi.org/10.1016/j.nbt.2016.05.001

9. Obruca, S., Sedlacek, P., Koller, M., Kucera, D., Pernico$v a, I$., Involvement of polyhydroxyalkanoates in stress resistance of microbial cells: Biotechnological consequences and applications, Biotechnol. Adv. 36 (2018) 856. doi: https://doi.org/10.1016/j.biotechadv.2017.12.006

10. Slaninova, E., Sedlacek, P., Mravec, F., Mullerova, L., Samek, O., Koller, M., Hesko, O., Kucera, D., Marova, I., Obruca, S., Light scattering on PHA granules protects bacterial cells against the harmful effects of UV radiation, Appl. Microbiol. Biotechnol. 102 (2018) 1923. doi: https://doi.org/10.1007/s00253-018-8760-8

11. Obruca, S., Marova, I., Stankova, M., Mravcova, L., Svoboda, Z., Effect of ethanol and hydrogen peroxide on poly (3-hydroxybutyrate) biosynthetic pathway in Cupriavidus necator H16, World J. Microbiol. Biotechnol. 26 (2010) 1261. doi: https://doi.org/10.1007/s11274-009-0296-8

12. Obruca, S., Sedlacek, P., Mravec, F., Samek, O., Marova, $I$., Evaluation of 3-hydroxybutyrate as an enzyme-protective agent against heating and oxidative damage and its potential role in stress response of poly(3-hydroxybutyrate) accumulating cells, Appl. Microbiol. Biotechnol. 100 (2016) 1365. doi: https://doi.org/10.1007/s00253-015-7162-4

13. Obruca, S., Sedlacek, P., Mravec, F., Krzyzanek, V., Nebesarova, J., Samek, O., Kucera, D., Benesova, P., Hrubanova, K., Milerova, M., Marova, I., The presence of PHB granules in cytoplasm protects non-halophilic bacterial cells against the harmful impact of hypertonic environments, New Biotechnol. 39 (2017) 68. doi: https://doi.org/10.1016/j.nbt.2017.07.008

14. Obruca, S., Sedlacek, P., Krzyzanek, V., Mravec, F., Hrubanova, K., Samek, O., Kucera, D., Benesova, P., Marova, I., Accumulation of poly(3-hydroxybutyrate) helps bacterial cells to survive freezing, PloS one 11 (2016) e0157778.

doi: https://doi.org/10.1371/journal.pone.0157778 
15. Jiang, G., Hill, D. J., Kowalczuk, M., Johnston, B., Adamus, G., Irorere, V., Radecka, I., Carbon sources for polyhydroxyalkanoates and an integrated biorefinery, Int. J. Mol. Sci. 17 (2016) 1157 doi: https://doi.org/10.3390/ijms17071157

16. Dietrich, K., Dumont, M. J., Del Rio, L. F., Orsat, V., Producing PHAs in the bioeconomy-Towards a sustainable bioplastic, Sus. Prod. Cons. 9 (2017) 58. doi: https://doi.org/10.1016/j.spc.2016.09.001

17. Shahzad, K., Narodoslawsky, M., Sagir, M., Ali, N., Ali, S., Rashid, M. I., Ismail, I. M. I., Koller, M., Techno-economic feasibility of waste biorefinery: Using slaughtering waste streams as starting material for biopolyester production, Waste Manage. 67 (2017) 73. doi: https://doi.org/10.1016/j.wasman.2017.05.047

18. Obruca, S., Benesova, P., Marsalek, L., Marova, I. Use of lignocellulosic materials for PHA production, Chem. Biochem. Eng. Q. 29 (2015) 135. doi: https://doi.org/10.15255/CABEQ.2014.2253

19. Koller, M., Atlić, A., Dias, M., Reiterer, A., Braunegg, G., Microbial PHA Production from Waste Raw Materials, in Chen, G.-Q. (Ed.), Plastics from Bacteria Springer, Berlin, Heidelberg, 2010, pp 85-119. doi: https://doi.org/10.1007/978-3-642-03287-5_5

20. Albuquerque, M. G. E., Eiroa, M., Torres, C., Nunes, B. R., Reis, M. A. M., Strategies for the development of a side stream process for polyhydroxyalkanoate (PHA) production from sugar cane molasses, J. Biotechnol. 130 (2007) 411 doi: https://doi.org/10.1016/j.jbiotec.2007.05.011

21. Pittmann, T., Steinmetz, H., Polyhydroxyalkanoate production on waste water treatment plants: Process scheme, operating conditions and potential analysis for German and European municipal waste water treatment plants, Bioengineering 4 (2017) 54. doi: https://doi.org/10.3390/bioengineering 4020054

22. Queirós, D., Rossetti, S., Serafim, L. S., PHA production by mixed cultures: A way to valorize wastes from pulp industry, Biores. Technol. 157 (2014) 197. doi: https://doi.org/10.1016/j.biortech.2014.01.099

23. Koller, M., Sandholzer, D., Salerno, A., Braunegg, G., Narodoslawsky, M., Biopolymer from industrial residues: Life cycle assessment of poly(hydroxyalkanoates) from whey, Resour. Conserv. Recy. 73 (2013) 64. doi: https://doi.org/10.1016/j.resconrec.2013.01.017

24. Kourmentza, C., Koutra, E., Venetsaneas, N., Kornaros, M., Integrated Biorefinery Approach for the Valorization of Olive Mill Waste Streams Towards Sustainable Biofuels and Bio-Based Products, in Kalia, V., Kumar, P. (Eds.), Microbial Applications, Vol. 1, Springer, Cham, 2017, pp 211-238.

doi: https://doi.org/10.1007/978-3-319-52666-9_10

25. Troschl, C., Meixner, K., Drosg, B., Cyanobacterial PHA production - Review of recent advances and a summary of three years' working experience running a pilot plant, Bioengineering 4 (2017) 26. doi: https://doi.org/10.3390/bioengineering4020026

26. Khosravi-Darani, K., Mokhtari, Z. B., Amai, T., Tanaka, $K$., Microbial production of poly(hydroxybutyrate) from C carbon sources, Appl. Microbiol. Biotechnol. 97 (2013) 1407.

doi: https://doi.org/10.1007/s00253-012-4649-0

27. Tanaka, K., Miyawaki, K., Yamaguchi, A., Khosravi-Darani, $K$., Matsusaki, H., Cell growth and $\mathrm{P}(3 \mathrm{HB})$ accumulation from $\mathrm{CO}_{2}$ of a carbon monoxide-tolerant hydrogen-oxidizing bacterium, Ideonella sp. O-1, Appl. Microbiol. Biotechnol. 92 (2011) 1161. doi: https://doi.org/10.1007/s00253-011-3420-2
28. Mokhtari-Hosseini, Z. B., Vasheghani Farahani, E., Shojaosadati, S. A., Karimzadeh, R., Khosravi-Darani, K., Media selection for poly (hydroxybutyrate) production from methanol by Methylobacterium extorquens DSMZ 1340, Iran. J. Chem. Chem. Eng. 28 (2009) 45.

29. Mokhtari-Hosseini, Z. B., Vasheghani-Farahani, E., Heidarzadeh-Vazifekhoran, A., Shojaosadati, S. A., Karimzadeh, R., Khosravi-Darani, K., Statistical media optimization for growth and PHB production from methanol by a methylotrophic bacterium, Biores. Technol. 100 (2009) 2436 doi: https://doi.org/10.1016/j.biortech.2008.11.024

30. Karmann, S., Follonier, S., Egger, D., Hebel, D., Panke, S., Zinn, M., Tailor-made PAT platform for safe syngas fermentations in batch, fed-batch and chemostat mode with Rhodospirillum rubrum, Microb. Biotechnol. 10 (2017) 1365.

doi: https://doi.org/10.1111/1751-7915.12727

31. Koller, M., Niebelschütz, H., Braunegg, G., Strategies for recovery and purification of poly $[(R)$-3-hydroxyalkanoates](PHA) biopolyesters from surrounding biomass. Eng. Life Sci. 13 (2013) 549. doi: https://doi.org/10.1002/elsc.201300021

32. Kosseva, M. R., Rusbandi, E., Trends in the biomanufacture of polyhydroxyalkanoates with focus on downstream processing, Int. J. Biol. Macromol. 107 (2018) 762. doi: https://doi.org/10.1016/j.ijbiomac.2017.09.054

33. Madkour, M. H., Heinrich, D., Alghamdi, M. A., Shabbaj, I. I., Steinbüchel, A., PHA recovery from biomass, Biomacromolecules 14 (2013) 2963.

34. Daly, S. R., Fathi, A., Bahramian, B., Manavitehrani, I., McClure, D. D., Valtchev, P., Schindeler, A., Dehghani, F., Kavanagh, J. M., A green process for the purification of biodegradable poly( $\beta$-hydroxybutyrate), J. Supercrit. Fluid. 135 (2018) 84 doi: https://doi.org/10.1016/j.supflu.2018.01.007

35. Khosravi-Darani, K., Vasheghani-Farahani, E., Shojaosadati, S. A., Yamini, Y., Effect of process variables on supercritical fluid disruption of Ralstonia eutropha cells for poly( $R$-hydroxybutyrate) recovery, Biotechnol. Prog. 20 (2004) 1757. doi: https://doi.org/10.1021/bp0498037

36. Khosravi-Darani, K., Vasheghani-Farahani, E., Yamini, $Y$., Bahramifar, N., Solubility of poly( $\beta$-hydroxybutyrate) in supercritical carbon dioxide, J. Chem. Eng. Data 48 (2003) 860 . doi: https://doi.org/10.1021/je020168v

37. Khosravi-Darani, K., Vasheghani-Farahani, E., Shojaosadati, S. A., Yamini, Y., Effect of process variables on supercritical fluid disruption of Ralstonia eutropha cells for poly $(R$-hydroxybutyrate) recovery, Biotechnol. Prog. 20 (2004) 1757. doi: https://doi.org/10.1021/10.1021/bp0498037

38. Koller, M., Bona, R., Chiellini, E., Braunegg, G., Extraction of short-chain-length poly- $[(R)$-hydroxyalkanoates] (scl-PHA) by the "anti-solvent" acetone under elevated temperature and pressure, Biotechnol. Lett. 35 (2013) 1023. doi: https://doi.org/10.1007/s10529-013-1185-7

39. Dubey, S., Bharmoria, P., Gehlot, P. S., Agrawal, V., Kumar, A., Mishra, S., 1-Ethyl-3-methylimidazolium diethylphosphate based extraction of bioplastic "Polyhydroxyalkanoates" from bacteria: Green and sustainable approach, ACS Sustain. Chem. Eng. 6 (2017) 766. doi: https://doi.org/10.1021/acssuschemeng.7b03096 
40. Ong, S. Y., Zainab-L, I., Pyary, S., Sudesh, K., A novel biological recovery approach for PHA employing selective digestion of bacterial biomass in animals, Appl. Microbiol. Biotechnol. 102 (2018) 2177. doi: https://doi.org/10.1007/s00253-018-8788-9

41. Gamero, J. E. R., Fava, L., Pizzocchero, V., P., Lomolino, G., Basaglia, M., Casella, S., Nuclease expression in efficient polyhydroxyalkanoates-producing bacteria could yield cost reduction during downstream processing, Biores. Technol. 261 (2018) 176. doi: https://doi.org/10.1016/j.biortech.2018.04.021

42. Hsiao, L. J., Lee, M. C., Chuang, P. J., Kuo, Y. Y., Lin, J. $H$., $W u, T . M$., Li, S. Y., The production of poly(3-hydroxybutyrate) by thermophilic Caldimonas manganoxidans from glycerol, J. Polym. Res. 25 (2018) 85. doi: https://doi.org/10.1007/s10965-018-1486-6

43. Koller, M., Production of polyhydroxyalkanoate (PHA) biopolyesters by extremophiles, MOJ Polym. Sci. 1 (2017) 1 . doi: https://doi.org/10.15406/mojps.2017.01.00011

44. Kucera, D., Pernicová, I., Kovalcik, A., Koller, M., Mullerova, L., Sedlacek, P., Mravec, F., Nebesarova, J., Kalina, M., Marova, I., Krzyzanek, V., Obruca, S., Characterization of the promising poly(3-hydroxybutyrate) producing halophilic bacterium Halomonas halophila, Biores. Technol. 256 (2018) 552 doi: https://doi.org/10.1016/j.biortech.2018.02.062

45. Rodriguez-Contreras, A., Koller, M., Braunegg, G., Marqués-Calvo, M. S., Poly[(R)-3-hydroxybutyrate] production under different salinity conditions by a novel Bacillus megaterium strain, New Biotechnol. 33 (2016) 73. doi: https://doi.org/10.1016/j.nbt.2015.08.006

46. Yin, J., Chen, J. C., Wu, Q., Chen, G.-Q., Halophiles, coming stars for industrial biotechnology, Biotechnol. Adv. 33 (2015) 1433. doi: https://doi.org/10.1016/j.biotechadv.2014.10.008

47. Koller, M., A Review on established and emerging fermentation schemes for microbial production of polyhydroxyalkanoate (PHA) biopolyesters, Fermentation 4 (2018) 30. doi: https://doi.org/10.3390/fermentation4020030

48. Chen, G.-Q., Hajnal, I., The 'PHAome', Trends Biotechnol. 33 (2015) 559. doi: https://doi.org/10.1016/j.tibtech.2015.07.006

49. Rehm, B. H., Bacterial polymers: Biosynthesis, modifications and applications, Nat. Rev. Microbiol. 8 (2010) 578. doi: https://doi.org/10.1038/nrmicro2354

50. Braunegg, G., Lefebvre, G., Genser, K. F., Polyhydroxyalkanoates, biopolyesters from renewable resources: Physiological and engineering aspects, J. Biotechnol. 65 (1998) 127

doi: https://doi.org/10.1016/S0168-1656(98)00126-6

51. Koller, M., Salerno, A., Braunegg, G., Polyhydroxyalkanoates: Basics, Production and Applications of Microbial Biopolyesters, in Kabasci, St. (Ed.) Bio-based plastics: materials and applications, Wiley-VCH, Weinheim, 2013, pp. 137-170. doi: https://doi.org/10.1002/9781118676646.ch7

52. Rehm, B. H., Polyester synthases: Natural catalysts for plastics, Biochem. J. 376 (2003) 15. doi: https://doi.org/10.1042/bj20031254

53. Aneja, P., Charles, T. C., Poly-3-hydroxybutyrate degradation in Rhizobium (Sinorhizobium) meliloti: Isolation and characterization of a gene encoding 3-hydroxybutyrate dehydrogenase, J. Bacteriol. 181 (1999) 849. doi:https://doi.org/0021-9193/99/\$04.0010
54. Doi, Y., Segawa, A., Kawaguchi, Y., Kunioka, M., Cyclic nature of poly(3-hydroxyalkanoate) metabolism in $\mathrm{Al}$ caligenes eutrophus, FEMS Microbiol. Lett. 67 (1990) 165 . doi: https://doi.org/10.1111/j.1574-6968.1990.tb13856.x

55. Adaya, L., Millán, M., Peña, C., Jendrossek, D., Espín, G., Tinoco-Valencia, R., Guzman, J., Pfeiffer, D., Segura, D., Inactivation of an intracellular poly-3-hydroxybutyrate depolymerase of Azotobacter vinelandii allows to obtain a polymer of uniform high molecular mass, Appl. Microbiol. Biotechnol. 102 (2018) 2693. doi: https://doi.org/10.1007/s00253-018-8806-y

56. Kusaka, S., Iwata, T., Doi, Y., Properties and biodegradability of ultra-high-molecular-weight poly[(R)-3-hydroxybutyrate] produced by a recombinant Escherichia coli, Int. J. Biol. Macromol. 25 (1999) 87. doi: https://doi.org/10.1016/S0141-8130(99)00019-7

57. Elbanna, K., Lütke-Eversloh, T., Jendrossek, D., Luftmann, H., Steinbüchel, A., Studies on the biodegradability of polythioester copolymers and homopolymers by polyhydroxyalkanoate (PHA)-degrading bacteria and PHA depolymerases, Arch. Microbiol. 182 (2004) 212. doi: https://doi.org/10.1007/s00203-004-0715-z

58. Williams, M., Applications of PHAs in Medicine and Pharmacy, in Doi, Y., A. Steinbüchel, A. (Eds.), Biopolymers 4, Polyesters III - Applications, Wiley-VCH, Weinheim, 2002, pp 91-103.

59. Zinn, M., Biosynthesis of Medium-chain-length Poly $[(R)-$ 3-hydroxyalkanoates], in Chen, G.-Q., Steinbüchel, A. (Eds.), Plastics from Bacteria, Natural Functions and Applications, Springer, Münster, 2010, pp. 213-236. doi: https://doi.org/10.1007/978-3-642-03287-5_9

60. Vandamme, P., Coenye, T., Taxonomy of the genus Cupriavidus: A tale of lost and found, Int. J. Syst. Evol. Microbiol. 54 (2004) 2285. doi: https://doi.org/10.1099/ijs.0.63247-0

61. Poehlein, A., Kusian, B., Friedrich, B., Daniel, R., Bowien, $B$., Complete genome sequence of the type strain $\mathrm{Cu}$ priavidus necator N-1, J. Bacteriol. 193 (2011) 5017. doi: https://doi.org/10.1128/JB.05660-11

62. Mifune, J., Nakamura, S., Fukui, T., Engineering of pha operon on Cupriavidus necator chromosome for efficient biosynthesis of poly(3-hydroxybutyrate-co-3-hydroxyhexanoate) from vegetable oil, Polym. Degrad. Stab. 95 (2010) 1305. doi: https://doi.org/10.1016/j.polymdegradstab.2010.02.026

63. Kunioka, M., Kawaguchi, Y., Doi, Y., Production of biodegradable copolyesters of 3-hydroxybutyrate and 4-hydroxybutyrate by Alcaligenes eutrophus, Appl. Microbial. Biotechnol. 30 (1989) 569. doi: https://doi.org/10.1007/BF00255361

64. Cong, C., Zhang, S., Xu, R., Lu, W., Yu, D., The influence of $4 \mathrm{HB}$ content on the properties of poly(3-hydroxylbutyrate-co-4-hydroxylbutyrate) based on melt molded sheets, J. Appl. Polym. Sci. 109 (2008) 1962. doi: https://doi.org/10.1002/app.27873

65. Wen, X., Lu, X., Peng, Q., Zhu, F., Zheng, N., Crystallization behaviors and morphology of biodegradable poly(3-hydroxybutyrate-co-4-hydroxybutyrate), J. Therm. Anal. Calorim. 109 (2012) 959. doi: https://doi.org/10.1007/s10973-011-1768-2

66. Doi, Y., Kanesawa, Y., Kunioka, M., Saito, T., Biodegradation of microbial copolyesters: poly(3-hydroxybutyrate-co-3-hydroxyvalerate) and poly(3-hydroxybutyrate-co-4-hydroxybutyrate), Macromolecules 23 (1990) 26. 
67. Türesin, F., Gürsel, I., Hasirci, V., Biodegradable polyhydroxyalkanoate implants for osteomyelitis therapy: In vitro antibiotic release, J. Biomat. Sci. Polym. Ed. 12 (2001) 195. doi: https://doi.org/10.1163/156856201750180924

68. Martin, D. P., Williams, S. F., Medical applications of poly-4-hydroxybutyrate: A strong flexible absorbable biomaterial, Biochem. Eng. J. 16 (2003) 97. doi: https://doi.org/10.1016/S1369-703X(03)00040-8

69. Brigham, C. J., Sinskey, A. J., Applications of polyhydroxyalkanoates in the medical industry, Int. J. Biotechnol. Wellness Ind. 1 (2012) 52. doi: https://doi.org/10.6000/1927-3037.2012.01.01.03

70. Cesário, M. T., Raposo, R. S., de Almeida, M. C. M., Van Keulen, F., Ferreira, B. S., Telo, J. P., da Fonseca, M. M. $R$., Production of poly(3-hydroxybutyrate-co-4-hydroxybutyrate) by Burkholderia sacchari using wheat straw hydrolysates and gamma-butyrolactone, Int. J. Biol. Macromol. 71 (2014) 59. doi: https://doi.org/10.1016/j.ijbiomac.2014.04.054

71. Miranda De Sousa Dias, M., Koller, M., Puppi, D., Morelli, A., Chiellini, F., Braunegg, G., Fed-batch synthesis of poly(3-hydroxybutyrate) and poly(3-hydroxybutyrate-co-4-hydroxybutyrate) from sucrose and 4-hydroxybutyrate precursors by Burkholderia sacchari strain DSM 17165, Bioengineering 4 (2017) 36. doi: https://doi.org/10.3390/bioengineering4020036

72. Cavalheiro, J. M., Raposo, R. S., de Almeida, M. C. M., Cesário, M. T., Sevrin, C., Grandfils, C., Da Fonseca, M. $M$. R., Effect of cultivation parameters on the production of poly(3-hydroxybutyrate-co-4-hydroxybutyrate) and poly(3-hydroxybutyrate-4-hydroxybutyrate-3-hydroxyvalerate) by Cupriavidus necator using waste glycerol, Biores. Technol. 111 (2012) 391. doi: https://doi.org/10.1016/j.biortech.2012.01.176

73. Lee, Y. H., Kang, M. S., Jung, Y. M., Regulating the molar fraction of 4-hydroxybutyrate in poly(3-hydroxybutyrate-4-hydroxybutyrate) biosynthesis by Ralstonia eutropha using propionate as a stimulator, J. Biosci. Bioeng. 89 (2000) 380 doi: https://doi.org/10.1016/S1389-1723(00)88963-X

74. Hermann-Krauss, C., Koller, M., Muhr, A., Fasl, H., Stelzer, F., Braunegg, G., Archaeal production of polyhydroxyalkanoate (PHA) co-and terpolyesters from biodiesel industry-derived by-products, Archaea 2013 (2013) article ID 129268 doi: https://doi.org/10.1155/2013/129268

75. Aziz, N. A., Huong, K. H., Sipaut, C. S., Amirul, A. A., A fed-batch strategy to produce high poly(3-hydroxybutyrate-co-3-hydroxyvalerate-co-4-hydroxybutyrate) terpolymer yield with enhanced mechanical properties in bioreactor, Bioproc. Biosyst. Eng. 40 (2017) 1643. doi: https://doi.org/10.1007/s00449-017-1820-0

76. Chen, X., Yin, J., Ye, J., Zhang, H., Che, X., Ma, Y., Li, M., Wu, L.-P., Chen, G.-Q., Engineering Halomonas bluephagenesis TD01 for non-sterile production of poly(3-hydroxybutyrate-co-4-hydroxybutyrate), Biores. Technol. 244 (2017) 534. doi: https://doi.org/10.1016/j.biortech.2017.07.149

77. Jianwen, Y., Dingkai, H., Xuemei, C., Xiaoran, J., Teng, L., Jinchun, C., Zhang, H. M., Chen, G.-Q., Engineering of Halomonas bluephagenesis for low cost production of poly(3-hydroxybutyrate-co-4-hydroxybutyrate) from glucose, Metab. Eng. 47 (2018) 143. doi: https://doi.org/10.1016/j.ymben.2018.03.013
78. Ye, J., Huang, W., Wang, D., Chen, F., Yin, J., Li, T., Zhang, H., Chen, G.-Q., Pilot scale-up of poly(3-hydroxybutyrate-co-4-hydroxybutyrate) production by Halomonas bluephagenesis via cell growth adapted optimization process, Biotechnol. J. 13 (2018) 1800074. doi: https://doi.org/10.1002/biot.201800074

79. Ren, Y., Ling, C., Hajnal, I., Wu, Q., Chen, G.-Q., Construction of Halomonas bluephagenesis capable of high cell density growth for efficient PHA production, Appl. Microbiol. Biotechnol. 102 (2018) 4499. doi: https://doi.org/10.1007/s00253-018-8931-7

80. Lv, L., Ren, Y. L., Chen, J. C., Wu, Q., Chen, G.-Q., Application of CRISPRi for prokaryotic metabolic engineering involving multiple genes, a case study: Controllable P(3HB-co-4HB) biosynthesis, Metab. Eng. 29 (2015) 160. doi: https://doi.org/10.1016/j.ymben.2015.03.013

81. Lütke-Eversloh, T., Bergander, K., Luftmann, H., Steinbüchel, A., Identification of a new class of biopolymer: Bacterial synthesis of a sulfur-containing polymer with thioester linkages, Microbiology 147 (2001) 11.

82. Wübbeler, J. H., Steinbüchel, A., New pathways for bacterial polythioesters, Curr. Opin. Biotechnol. 29 (2014) 85. doi: https://doi.org/10.1016/j.copbio.2014.02.017

83. Kim, D. Y., Lütke-Eversloh, T., Elbanna, K., Thakor, N., Steinbüchel, A., Poly(3-mercaptopropionate): A nonbiodegradable biopolymer?, Biomacromolecules 6 (2005) 897. doi: https://doi.org/10.1021/bm049334x

84. De Koning, G. J. M., Van Bilsen, H. M. M., Lemstra, P. J., Hazenberg, W., Witholt, B., Preusting, H., van der Galiën, $J$. G., Schirmer, A., Jendrossek, D., A biodegradable rubber by crosslinking poly(hydroxyalkanoate) from Pseudomonas oleovorans, Polymer 35 (1994) 2090. doi: https://doi.org/10.1016/0032-3861(94)90233-X

85. Rehm, B. H., Steinbüchel, A., Biochemical and genetic analysis of PHA synthases and other proteins required for PHA synthesis, Int. J. Biol. Macromol. 25 (1999) 3. doi: https://doi.org/10.1016/S0141-8130(99)00010-0

86. Rodrigues, P. R., Druzian, J. I., Impact of different bacterial strains on the production, composition, and properties of novel polyhydroxyalkanoates using crude palm oil as substrate, Chem. Biochem. Eng. Q. 32 (2018) 141. doi: https://doi.org/10.15255/CABEQ.2017.1207

87. De Waard, P., Van der Wal, H., Huijberts, G. N., Eggink, $G$., Heteronuclear NMR analysis of unsaturated fatty acids in poly(3-hydroxyalkanoates). Study of beta-oxidation in Pseudomonas putida, J. Biol. Chem. 268 (1993) 315.

88. Muhr, A., Rechberger, E. M., Salerno, A., Reiterer, A., Schiller, M., Kwiecień, M., Adamus, G., Kowalczuk, M., Strohmeier, K., Schober, S., Mittelbach, M., Koller, M., Biodegradable latexes from animal-derived waste: Biosynthesis and characterization of $m c l$-PHA accumulated by P. citronellolis, React. Funct. Polym. 73 (2013) 1391. doi: https://doi.org/10.1016/j.reactfunctpolym.2012.12.009

89. Muhr, A., Rechberger, E. M., Salerno, A., Reiterer, A., Malli, K., Strohmeier, K., Schober, S., Mittelbach, M., Koller, $M$., Novel description of $\mathrm{mcl}$-PHA biosynthesis by Pseudomonas chlororaphis from animal-derived waste, J. Biotechnol. 165 (2013) 45. doi: https://doi.org/10.1016/j.jbiotec.2013.02.003

90. Liu, W., Chen, G.-Q., Production and characterization of medium-chain-length polyhydroxyalkanoate with high 3-hydroxytetradecanoate monomer content by $\mathrm{fadB}$ and fadA knockout mutant of Pseudomonas putida KT2442, Appl. Microbiol. Biotechnol. 76 (2007) 1153. doi: https://doi.org/10.1007/s00253-007-1092-8 
91. Huijberts, G. N., Eggink, G., De Waard, P., Huisman, G. W., Witholt, B., Pseudomonas putida KT2442 cultivated on glucose accumulates poly(3-hydroxyalkanoates) consisting of saturated and unsaturated monomers, Appl. Environ. Microbiol. 58 (1992) 536.

92. Sathiyanarayanan, G., Bhatia, S. K., Song, H. S., Jeon, J. M., Kim, J., Lee, Y. K., Kim, Y.-G., Yang, Y.-H., Production and characterization of medium-chain-length polyhydroxyalkanoate copolymer from Arctic psychrotrophic bacterium Pseudomonas sp. PAMC 28620, Int. J. Biol. Macromol. 97 (2017) 710.

doi: https://doi.org/10.1016/j.ijbiomac.2017.01.053

93. Muangwong, A., Boontip, T., Pachimsawat, J., Napathorn, $S$. $C$., Medium chain length polyhydroxyalkanoates consisting primarily of unsaturated 3-hydroxy-5-cis-dodecanoate synthesized by newly isolated bacteria using crude glycerol, Microb. Cell Fact. 15 (2016) 55 doi: https://doi.org/10.1186/s12934-016-0454-2

94. Mendonça, T. T., Tavares, R. R., Cespedes, L. G., Sánchez-Rodriguez, R. J., Schripsema, J., Taciro, M. K., Gomez, J. G. C., Silva, L. F., Combining molecular and bioprocess techniques to produce poly(3-hydroxybutyrate-co-3-hydroxyhexanoate) with controlled monomer composition by Burkholderia sacchari, Int. J. Biol. Macromol. 98 (2017) 654 doi: https://doi.org/10.1016/j.ijbiomac.2017.02.013

95. Zinn, M., Witholt, B., Egli, T., Dual nutrient limited growth: Models, experimental observations, and applications, J. Biotechnol. 113 (2004) 263. doi: https://doi.org/10.1016/j.jbiotec.2004.03.030

96. Hartmann, R., Hany, R., Geiger, T., Egli, T., Witholt, B., Zinn, M., Tailored biosynthesis of olefinic medium-chain-length Poly[(R)-3-hydroxyalkanoates] in Pseudomonas putida GPol with improved thermal properties, Macromolecules 37 (2004) 6780 doi: https://doi.org/10.1016/10.1021/ma040035+

97. Hany, R., Brinkmann, M., Ferri, D., Hartmann, R., Pletscher, E., Rentsch, D., Zinn, M., Crystallization of an aromatic biopolyesters, Macromolecules 42 (2009) 6322. doi: https://doi.org/ 10.1021/ma900995w

98. Barbuzzi, T., Giuffrida, M., Impallomeni, G., Carnazza, S., Ferreri, A., Guglielmino, S. P., Ballistreri, A., Microbial synthesis of poly(3-hydroxyalkanoates) by Pseudomonas aeruginosa from fatty acids: Identification of higher monomer units and structural characterization, Biomacromolecules 5 (2004) 2469.

doi: https://doi.org/10.1021/bm0495831

99. Ribeiro, P. L. L., da Silva, A. C. M. S., Menezes Filho, J. A., Druzian, J. I., Impact of different by-products from the biodiesel industry and bacterial strains on the production, composition, and properties of novel polyhydroxyalkanoates containing achiral building blocks, Ind. Crop. Prod. 69 (2015) 212. doi: https://doi.org/10.1016/j.indcrop.2015.02.035

100. Impallomeni, G., Ballistreri, A., Carnemolla, G. M., Rizzo, M. G., Nicolo, M. S., Guglielmino, S., Biosynthesis and structural characterization of polyhydroxyalkanoates produced by Pseudomonas aeruginosa ATCC 27853 from long odd-chain fatty acids, Int. J. Biol. Macromol. 108 (2018) 608 doi: https://doi.org/10.1016/j.jibiomac.2017.12.037

101. Ray, S., Kalia, V. C., Co-metabolism of substrates by $B a-$ cillus thuringiensis regulates polyhydroxyalkanoate co-polymer composition, Biores. Technol. 224 (2017) 743. doi: https://doi.org/10.1016/j.biortech.2016.11.089

102. Guo, W., Duan, J., Geng, W., Feng, J., Wang, S., Song, C., Comparison of medium-chain-length polyhydroxyal- kanoates synthases from Pseudomonas mendocina NK-01 with the same substrate specificity, Microbiol. Res. 168 (2013) 231 doi: https://doi.org/10.1016/j.micres.2012.11.003

103. Lenz, R. W., Marchessault, R. H., Bacterial polyesters: Biosynthesis, biodegradable plastics and biotechnology, Biomacromolecules 6 (2005) 1. doi: https://doi.org/10.1021/bm049700c

104. Koller, M., Bona, R., Hermann, C., Horvat, P., Martinz, J., Neto, J., Pereira, L., Varila, P., Braunegg, G., Biotechnological production of poly(3-hydroxybutyrate) with $\mathrm{Wau}$ tersia eutropha by application of green grass juice and silage juice as additional complex substrates, Biocat. Biotrans. 23 (2005) 329. doi: https://doi.org/10.1080/10242420500292252

105. Wallen, L. L., Rohwedder, W. K., Poly-beta-hydroxyalkanoate from activated sludge, Environ. Sci. Technol. 8 (1974) 576 doi: https://doi.org/10.1021/es60091a007

106. Findlay, R. H., White, D. C., Polymeric beta-hydroxyalkanoates from environmental samples and Bacillus megaterium, Appl. Environ. Microbiol. 45 (1983) 71.

107. Doi, Y., Tamaki, A., Kunioka, M., Soga, K., Production of copolyesters of 3-hydroxybutyrate and 3-hydroxyvalerate by Alcaligenes eutrophus from butyric and pentanoic acids, Appl. Microbiol. Biotechnol. 28 (1988) 330. doi: https://doi.org/10.1007/BF00268190

108. Lefebvre, G., Rocher, M., Braunegg, G., Effects of low dissolved-oxygen concentrations on poly-(3-hydroxybutyrate-co-3-hydroxyvalerate) production by Alcaligenes eutrophus, Appl. Environ. Microbiol. 63 (1997) 827.

109. Montano-Herrera, L., Laycock, B., Werker, A., Pratt, S., The evolution of polymer composition during PHA accumulation: The significance of reducing equivalents, Bioengineering 4 (2007) 20 . doi: https://doi.org/ 10.3390/bioengineering4010020

110. Koller, M., Bona, R., Chiellini, E., Fernandes, E. G., Horvat, P., Kutschera, C., Hesse, P., Braunegg, G., Polyhydroxyalkanoate production from whey by Pseudomonas hydrogenovora, Biores. Technol. 99 (2008) 4854. doi: https://doi.org/10.1016/j.biortech.2007.09.049

111. Koller, M., Hesse, P., Fasl, H., Stelzer, F., Braunegg, G., Study on the effect of levulinic acid on whey-based biosynthesis of poly(3-hydroxybutyrate-co-3-hydroxyvalerate) by Hydrogenophaga pseudoflava, Appl. Food Biotechnol. 4 (2017) 65. doi: https://doi.org/10.22037/afb.v4i2.16337

112. Ashby, R. D., Solaiman, D. K., Nuñez, A., Strahan, G. D., Johnston, D. B., Burkholderia sacchari DSM 17165: A source of compositionally-tunable block-copolymeric short-chain poly(hydroxyalkanoates) from xylose and levulinic acid, Biores. Technol. 253 (2018) 333. doi: https://doi.org/10.1016/j.biortech.2017.12.045

113. Koller, M., Miranda de Sousa Dias, M., Rodríguez-Contreras, A., Kunaver, M., Žagar, E., Kržan, A., Braunegg, $G$., Liquefied wood as inexpensive precursor-feedstock for bio-mediated incorporation of $(R)$-3-hydroxyvalerate into polyhydroxyalkanoates, Materials 8 (2015) 6543. doi: https://doi.org/10.3390/ma8095321

114. Koller, M., Salerno, A., Strohmeier, K., Schober, S., Mittelbach, M., Illieva, V., Chiellini, E., Braunegg, G., Novel precursors for production of 3-hydroxyvalerate-containing poly $[(R)$-hydroxyalkanoate $]$ s, Biocat. Biotrans. 32 (2014) 161.

doi: https://doi.org/10.3109/10242422.2014.913580 
115. Kumar, P., Ray, S., Kalia, V. C., Production of co-polymers of polyhydroxyalkanoates by regulating the hydrolysis of biowastes, Biores. Technol. 200 (2016) 413. doi: https://doi.org/10.1016/j.biortech.2015.10.045

116. Salgaonkar, B. B., Bragança, J. M., Biosynthesis of poly(3-hydroxybutyrate-co-3-hydroxyvalerate) by Halogeometricum borinquense strain E3, Int. J. Biol. Macromol. 78 (2015) 339.

doi: https://doi.org/10.1016/j.ijbiomac.2015.04.016

117. Salgaonkar, B. B., Bragança, J. M., Utilization of sugarcane bagasse by Halogeometricum borinquense strain E3 for biosynthesis of poly(3-hydroxybutyrate-co-3-hydroxyvalerate), Bioengineering 4 (2017) 50. doi: https://doi.org/10.3390/bioengineering4020050

118. Bhattacharya, S., Dubey, S., Singh, P., Shrivastava, A., Mishra, S., Biodegradable polymeric substances produced by a marine bacterium from a surplus stream of the biodiesel industry, Bioengineering 3 (2016) 34. doi: https://doi.org/10.3390/bioengineering3040034

119. Noda, I., Green, P. R., Satkowski, M. M., Schechtman, L. $A$., Preparation and properties of a novel class of polyhydroxyalkanoate copolymers, Biomacromolecules 6 (2015) 580 doi: https://doi.org/10.1021/bm049472m

120. Nakamura, S., Kunioka, M., Doi, Y., Biosynthesis and characterization of bacterial poly(3-hydroxybutyrate-co-3-hydroxypropionate), Macromol. Rep. 28 (1991) 15.

121. Hiramitsu, M., Doi, Y., Microbial synthesis and characterization of poly(3-hydroxybutyrate-co-3-hydroxypropionate), Polymer 34 (1993) 4782.

122. Shimamura, E., Scandola, M., Doi, Y., Microbial synthesis and characterization of poly(3-hydroxybutyrate-co-3-hydroxypropionate), Macromolecules 27 (1994) 4429.

123. Andreeßen, B., Steinbüchel, A., Biosynthesis and biodegradation of 3-hydroxypropionate-containing polyesters, Appl. Environ. Microbiol. 76 (2010) 4919. doi: https://doi.org/10.1128/AEM.01015-10

124. Kang, C. K., Lee, H. S., Kim, J. H., Accumulation of PHA and its copolyesters by Methylobacterium sp. KCTC 0048. Biotechnol. Lett. 15 (1993) 1017. doi: https://doi.org/10.1007/BF00129929

125. Valentin, H. E., Mitsky, T. A., Mahadeo, D. A., Tran, M., Gruys, $K$. J., Application of a propionyl coenzyme A synthetase for poly(3-hydroxypropionate-co-3-hydroxybutyrate) accumulation in recombinant Escherichia coli, Appl. Environ. Microb. 66 (2000) 5253. doi: https://doi.org/10.1128/AEM.66.12.5253-5258.2000

126. Fukui, T., Suzuki, M., Tsuge, T., Nakamura, S., Microbial synthesis of poly $((R)$-3-hydroxybutyrate-co-3-hydroxypropionate) from unrelated carbon sources by engineered Cupriavidus necator, Biomacromolecules 10 (2009) 700. doi: https://doi.org/10.1021/bm801391j

127. Andreeßen, B., Lange, A. B., Robenek, H., Steinbüchel, A., Conversion of glycerol to poly(3-hydroxypropionate) in recombinant Escherichia coli, Appl. Environ. Microbiol. 76 (2010) 622 doi: https://doi.org/10.1128/AEM.02097-09

128. Wang, Q., Yang, P., Liu, C., Xue, Y., Xian, M., Zhao, G., Biosynthesis of poly(3-hydroxypropionate) from glycerol by recombinant Escherichia coli, Biores. Technol. 131 (2013) 548. doi: https://doi.org/10.1016/j.biortech.2013.01.096

129. Wang, Q., Liu, C., Xian, M., Zhang, Y., Zhao, G., Biosynthetic pathway for poly(3-hydroxypropionate) in recombinant Escherichia coli, J. Microbiol. 50 (2012) 693. doi: https://doi.org/10.1007/s12275-012-2234-y
130. Watanabe, Y., Ishizuka, K., Furutate, S., Abe, H., Tsuge, T., Biosynthesis and characterization of novel poly(3-hydroxybutyrate-co-3-hydroxy-2-methylbutyrate): Thermal behavior associated with $\alpha$-carbon methylation, RSC Adv. 5 (2015) 58679. doi: https://doi.org/10.1039/C5RA08003G

131. Furutate, S., Nakazaki, H., Maejima, K., Hiroe, A., Abe, $H$., Tsuge, T., Biosynthesis and characterization of novel polyhydroxyalkanoate copolymers consisting of 3-hydroxy-2-methylbutyrate and 3-hydroxyhexanoate, J. Polym. Res. 24 (2017) 221. doi: https://doi.org/10.1007/s10965-017-1392-3

132. Dai, Y., Lambert, L., Yuan, Z., Keller, J., Characterisation of polyhydroxyalkanoate copolymers with controllable four-monomer composition, J. Biotechnol. 134 (2008) 137. doi: https://doi.org/10.1016/j.jbiotec.2008.01.013

133. Tanadchangsaeng, N., Tsuge, T., Abe, H., Comonomer compositional distribution, physical properties, and enzymatic degradability of bacterial poly(3-hydroxybutyrate-co-3-hydroxy-4-methylvalerate) copolyesters, Biomacromolecules 11 (2010) 1615. doi: https://doi.org/10.1021/bm100267k

134. Chia, K. H., Ooi, T. F., Saika, A., Tsuge, T., Sudesh, K., Biosynthesis and characterization of novel polyhydroxyalkanoate polymers with high elastic property by Cupriavidus necator $\mathrm{PHB}^{-} 4$ transformant, Polym. Degrad. Stab. 95 (2010) 2226. doi: https://doi.org/10.1016/j.polymdegradstab.2010.09.011

135. Ling, S. C., Tsuge, T., Sudesh, K., Biosynthesis of novel polyhydroxyalkanoate containing 3-hydroxy-4-methylvalerate by Chromobacterium sp. USM2. J. Appl. Microbiol. 111 (2011) 559. doi: https://doi.org/10.1111/j.1365-2672.2011.05084.x

136. Saika, A., Watanabe, Y., Sudesh, K., Abe, H., Tsuge, T., Enhanced incorporation of 3-hydroxy-4-methylvalerate unit into biosynthetic polyhydroxyalkanoate using leucine as a precursor, AMB express 1 (2011) 6. doi: https://doi.org/10.1186/2191-0855-1-6

137. Lau, N. S., Chee, J. Y., Tsuge, T., Sudesh, K., Biosynthesis and mobilization of a novel polyhydroxyalkanoate containing 3-hydroxy-4-methylvalerate monomer produced by Burkholderia sp. USM (JCM15050), Biores. Technol. 101 (2010) 7916. doi: https://doi.org/10.1016/j.biortech.2010.05.049

138. Kim, Y. B., Lenz, R. W., Fuller, R. C., Preparation and characterization of poly( $\beta$-hydroxyalkanoates) obtained from Pseudomonas oleovorans grown with mixtures of 5-phenylvaleric acid and $n$-alkanoic acids, Macromolecules 24 (1991) 5256.

139. Lenz, R. W., Kim, Y. B., Fuller, R. C., Production of unusual bacterial polyesters by Pseudomonas oleovorans through cometabolism, FEMS Microbiol. Rev. 9 (1992) 207. doi: https://doi.org/10.1111/j.1574-6968.1992.tb05839.x

140. Kim, Y. B., Lenz, R. W., Fuller, R. C., Poly( $\beta$-hydroxyalkanoate) copolymers containing brominated repeating units produced by Pseudomonas oleovorans, Macromolecules 25 (1992) 1852.

141. Doi, Y., Abe, C., Biosynthesis and characterization of a new bacterial copolyester of 3-hydroxyalkanoates and 3-hydroxy- $\omega$-chloroalkanoates, Macromolecules 23 (1990) 3705 .

142. Abe, C., Taima, Y., Nakamura, Y., Doi, Y., New bacterial copolyester of 3-hydroxyalkanoates and 3-hydroxy- $\omega$-fluoroalkanoates produced by Pseudomonas oleovorans, Polym. Commun. 31 (1990) 404. 
143. Hori, K., Soga, K., Doi, Y., Production of poly(3-hydroxyalkanoates-co-3-hydroxy- $\omega$-fluoroalkanoates) by Pseudomonas oleovorans from 1-fluorononane and gluconate, Biotechnol. Lett. 16 (1994) 501. doi: https://doi.org/10.1007/BF01023333

144. Bear, M. M., Leboucher-Durand, M. A., Langlois, V., Lenz, R. W., Goodwin, S., Guérin, P., Bacterial poly-3-hydroxyalkenoates with epoxy groups in the side chains, React. Funct. Polym. 34 (1997) 65. doi: https://doi.org/10.1016/S1381-5148(97)00024-2

145. Lee, M. Y., Park, W. H., Lenz, R. W., Hydrophilic bacterial polyesters modified with pendant hydroxyl groups, Polymer 41 (2000) 1703 doi: https://doi.org/10.1016/S0032-3861(99)00347-X

146. Kurth, N., Renard, E., Brachet, F., Robic, D., Guerin, P., Bourbouze, R., Poly(3-hydroxyoctanoate) containing pendant carboxylic groups for the preparation of nanoparticles aimed at drug transport and release, Polymer 43 (2002) 1095. doi: https://doi.org/10.1016/S0032-3861(01)00692-9

147. Scholz, C., Fuller, R. C., Lenz, R. W., Production of poly( $\beta$-hydroxyalkanoates) with $\beta$-substituents containing terminal ester groups by Pseudomonas oleovorans, Macromol. Chem. Physic. 195 (1994) 1405. doi: https://doi.org/10.1002/macp.1994.021950424

148. Zhila, N., Shishatskaya, E., Properties of PHA bi-, ter-, and quarterpolymers containing 4-hydroxybutyrate monomer units, Int. J. Biol. Macromol. 111 (2018) 1019. doi: https://doi.org/10.1016/j.ijbiomac.2018.01.130

149. Mizuno, S., Enda, Y., Saika, A., Hiroe, A., Tsuge, T., Biosynthesis of polyhydroxyalkanoates containing 2-hydroxy-4-methylvalerate and 2-hydroxy-3-phenylpropionate units from a related or unrelated carbon source, J. Biosci. Bioeng. 125 (2018) 295. doi: https://doi.org/10.1016/j.jbiosc.2017.10.010

150. Wang, H. H., Zhou, X. R., Liu, Q., Chen, G.-Q., Biosynthesis of polyhydroxyalkanoate homopolymers by Pseudomonas putida, Appl. Microbiol. Biotechnol. 89 (2011) 1497.

doi: https://doi.org/10.1007/s00253-010-2964-x

151. Liu, Q., Luo, G., Zhou, X. R., Chen, G.-Q., Biosynthesis of poly(3-hydroxydecanoate) and 3-hydroxydodecanoate dominating polyhydroxyalkanoates by $\beta$-oxidation pathway inhibited Pseudomonas putida, Metab. Eng. 13 (2011) 11. doi: https://doi.org/10.1016/j.ymben.2010.10.004

152. Chung, A. L., Jin, H. L., Huang, L. J., Ye, H. M., Chen, J. $C$., $W u, Q$., Chen, G.-Q., Biosynthesis and characterization of poly(3-hydroxydodecanoate) by $\beta$-oxidation inhibited mutant of Pseudomonas entomophila L48, Biomacromolecules 12 (2011) 3559. doi: https://doi.org/10.1021/bm200770m

153. Wang, Y., Chung, A., Chen, G.-Q., Synthesis of medium-chain-length polyhydroxyalkanoate homopolymers, random copolymers, and block copolymers by an engineered strain of Pseudomonas entomophila, Adv. Healthcare Mat. 6 (2017) 16010117. doi: https://doi.org/10.1002/adhm.201601017

154. Tripathi, L., Wu, L. P., Chen, J., Chen, G.-Q., Synthesis of Diblock copolymer poly-3-hydroxybutyrate-block-poly-3-hydroxyhexanoate [PHB- $b$-PHHx] by a $\beta$-oxidation weakened Pseudomonas putida KT2442, Microb. Cell Fact. 11 (2012) 44. doi: https://doi.org/10.1186/1475-2859-11-44

155. Pederson, E. N., McChalicher, C. W., Srienc, F., Bacterial synthesis of PHA block copolymers, Biomacromolecules 7 (2006) 1904

doi: https://doi.org/10.1021/bm0510101
156. Kawalec, M., Adamus, G., Kurcok, P., Kowalczuk, M., Synthesis of Poly $[(R, S)$-3-hydroxybutyrate-block-ethylene glycol-block- $(R, S)$-3-hydroxybutyrate] via anionic ROP, Macromol. Symp. 253 (2007) 59. doi: https://doi.org/10.1002/masy.200750707

157. Chen, C., Yu, C. H., Cheng, Y. C., Peter, H. F., Cheung, M. $K$., Biodegradable nanoparticles of amphiphilic triblock copolymers based on poly(3-hydroxybutyrate) and poly(ethylene glycol) as drug carriers, Biomaterials 27 (2006) 4804. doi: https://doi.org/10.1016/j.biomaterials.2006.04.039

158. Adamus, G., Sikorska, W., Janeczek, H., Kwiecień, M., Sobota, M., Kowalczuk, M., Novel block copolymers of atactic PHB with natural PHA for cardiovascular engineering: Synthesis and characterization, Eur. Polym. J. 48 (2012) 621. doi: https://doi.org/10.1016/j.eurpolymj.2011.12.017

159. Ferre-Guell, A., Winterburn, J., Biosynthesis and characterisation of polyhydroxyalkanoates with controlled composition and microstructure, Biomacromolecules 19 (2018) 996. doi: https:// 10.1021/acs.biomac.7b01788

160. Raposo, R. S., de Almeida, M. C. M., de Oliveira, M. D. C. M., da Fonseca, M. M., Cesário, M T., A Burkholderia sacchari cell factory: Pproduction of poly-3-hydroxybutyrate, xylitol and xylonic acid from xylose-rich sugar mixtures, New Biotechnol. 34 (2017) 12. doi: https://doi.org/10.1016/j.nbt.2016.10.001

161. Atlić, A., Koller, M., Scherzer, D., Kutschera, C., Grillo-Fernandes, E., Horvat, P., Chiellini, E., Braunegg, G., Continuous production of poly $([R]-3$-hydroxybutyrate) by Cupriavidus necator in a multistage bioreactor cascade, Appl. Microbiol. Biotechnol. 91 (2011) 295. doi: https://doi.org/10.1007/s00253-011-3260-0

162. Horvat, P., Špoljarić, I. V., Lopar, M., Atlić, A., Koller, M. Braunegg, G., Mathematical modelling and process optimization of a continuous 5-stage bioreactor cascade for production of poly[- $(R)-3$-hydroxybutyrate] by Cupriavidus necator, Bioproc. Biosys. Eng. 36 (2013) 1235. doi: https://doi.org/10.1007/s00449-012-0852-8

163. Lopar, M., Špoljarić, I. V., Atlić, A., Koller, M., Braunegg, G., Horvat, P., Five-step continuous production of PHB analyzed by elementary flux, modes, yield space analysis and high structured metabolic model, Biochem. Eng. J. 79 (2013) 57.

doi: https://doi.org/10.1016/j.bej.2013.07.003

164. Vadlja, D., Koller, M., Novak, M., Braunegg, G., Horvat, $P$., Footprint area analysis of binary imaged Cupriavidus necator cells to study PHB production at balanced, transient, and limited growth conditions in a cascade process, Appl. Microbiol. Biotechnol. 100 (2016) 10065. doi: https://doi.org/10.1007/s00253-016-7844-6

165. Koller, M., Vadlja, D., Braunegg, G., Atlić, A., Horvat, P. Formal- and high-structured kinetic process modelling and footprint area analysis of binary imaged cells: Tools to understand and optimize multistage-continuous PHA biosynthesis, The EuroBiotech Journal 1 (2017) 203. doi: https://doi.org/10.24190/ISSN2564-615X/2017/03.01

166. Ghysels, S., Mozumder, M. S. I., De Wever, H., Volcke, E. I., Garcia-Gonzalez, L., Targeted poly(3-hydroxybutyrate-co-3-hydroxyvalerate) bioplastic production from carbon dioxide, Biores. Technol. 249 (2018) 858. doi: https://doi.org/10.1016/j.biortech.2017.10.081

167. Cespedes, L. G., Nahat, R. A., Mendonça, T., Tavares, R. R., Oliveira-Filho, E. R., Silva, L. F., Taciro, M. K., Sanchez, R. J., Gomez, G. C. G., A non-naturally-occurring $\mathrm{P}(3 \mathrm{HB}-\mathrm{co}-3 \mathrm{HA} \mathrm{MCL})$ is produced by recombinant Pseudomonas sp. from an unrelated carbon source, Int. J. Biol. Macromol. 114 (2018) 512. doi: https://doi.org/10.1016/j.ijbiomac.2018.03.051 
168. Tripathi, L., Wu, L. P., Meng, D., Chen, J., Chen, G.-Q. Biosynthesis and characterization of diblock copolymer of $\mathrm{P}$ (3-hydroxypropionate)-block-P(4-hydroxybutyrate) from recombinant Escherichia coli, Biomacromolecules 14 (2013) 862. doi: https://doi.org/10.1021/bm3019517

169. Li, S. Y., Dong, C. L., Wang, S. Y., Ye, H. M., Chen, G.-Q., Microbial production of polyhydroxyalkanoate block copolymer by recombinant Pseudomonas putida, Appl. Microbiol. Biotechnol. 90 (2011) 659. doi: https://doi.org/10.1007/s00253-010-3069-2

170. Tripathi, L., Wu, L. P., Dechuan, M., Chen, J., Wu, Q., Chen, G.-Q., Pseudomonas putida KT2442 as a platform for the biosynthesis of polyhydroxyalkanoates with adjust- able monomer contents and compositions, Biores. Technol. 142 (2013) 225

doi: https://doi.org/10.1016/j.biortech.2013.05.027

171. Matsumoto, K. I., Hori, C., Fujii, R., Takaya, M., Ooba, T., Ooi, T., Isono, T., Satoh, T., Taguchi, S., Dynamic changes of intracellular monomer levels regulate block sequence of polyhydroxyalkanoates in engineered Escherichia coli, Biomacromolecules 19 (2018) 662. doi: https: doi.org/10.1021/acs.biomac.7b01768

172. Don, T. M., Chen, C. W., Chan, T. H., Preparation and characterization of poly(hydroxyalkanoate) from the fermentation of Haloferax mediterranei, J. Biomat. Sci. Polym. Ed. 17 (2006) 1425. doi: https: doi.org/10.1163/156856206778937208 